\title{
Fair Play in Assemblies
}

Michael Gibilisco*

University of Rochester, Department of Political Science, Rochester, New York,USA; michael.gibilisco@rochester.edu

\begin{abstract}
This paper studies the conditions under which minority proposal rights emerge from majority voting in a legislature. I develop a legislative bargaining model in which rules persist, i.e., remain in effect until a majority agrees to change them. In each session, legislators first determine whether a minority leader can offer amendments, and subsequently they determine policy using these procedures and majority rule. The main result demonstrates that legislative majorities grant minority rights today in order to moderate policy tomorrow when they may become the minority. This mechanism operates without punishment strategies and private information and in the presence of polarized and unified parties; however, persistent rules are necessary for the right to substantively influence policies. Comparative statics indicate that weak parties, super-majority rule, patient legislators, and extreme proposers encourage the adoption of minority rights. More broadly, these results demonstrate the importance of persistent rules for the endurance of inclusive institutions and political compromises, and they suggest one reason for procedural differences between the House and Senate.
\end{abstract}

${ }^{*}$ Thanks to Avi Acharya, Sergio Ascencio, Rob Carroll, Seok-Ju Cho, Mason Delang, John Duggan, Mark Fey, Gerald Gamm, Brenton Kenkel, Dave Primo, Mattan Sharkansky, Svanhildur Thorvaldsdottir, Yannis Vassiliadis, participants

Online Appendix available from: http://dx.doi.org/10.1561/100.00014155_app

MS submitted 6 November 2014; final version received 26 April 2015

ISSN 1554-0626; DOI 10.1561/100.00014155

(C) 2015 M. Gibilisco 
Although legislative agenda-setting powers entail substantial policy influence (Kalandrakis, 2006; McKelvey, 1976; Romer and Rosenthal, 1978; Shepsle and Weingast, 1987), majority coalitions often reserve some proposal rights for their minority counterparts. Legislatures frequently employ more open rules such as amendment agendas (Rasch, 1995) and allow members of the opposition to amend bills just prior to final passage (Krehbiel and Meirowitz, 2002; Mattson, 1995). Furthermore, the removal of these rights commonly requires a majority vote, so the rights must meet the tacit approval of either the legislative leadership or a legislative majority. Despite the prevalence and importance of minority proposal rights, political scientists lack a theoretical account of why majorities relinquish their agenda-setting monopoly under the most competitive conditions, including polarized voting coalitions and the absence of retaliation from future majorities or the electorate.

In this paper, I address this gap in the literature and advance a new theory of minority rights in legislatures where rules and policies persist, that is, continue from one legislative session to the next until a majority agrees to change them. Persistence does not mean rules and policies cannot change; indeed, legislators have the opportunity to alter them in every session. Rather it encourages legislators to consider the long-term consequences of their actions because those who prefer the current rules or policies receive a potential advantage tomorrow. For example, persistent rules reflect the interpretation of the U.S. Senate as a "continuing body" and hinder amending the cloture requirement even under a potential majority vote (Binder and Smith, 1997). Likewise, many western-democratic parliaments organize themselves using standing orders, a collection of rules which continue from session to session. ${ }^{1}$

To consider the effects procedural persistence, I construct a legislative bargaining model in which two parties, or coalitions of legislators, rotate between serving in the majority and minority. Each party has a

at MPSA and SPSA, and two anonymous referees for feedback and comments. In particular, thanks to Tasos Kalandrakis for invaluable discussions and encouragement. All errors are, naturally, my own.

${ }^{1}$ For example, Huber (1996) discusses how standing orders include important rules such as a vote of no confidence. In fact, the requirement that U.S. House of Representatives reaffirm its rules in every session before passing legislation is more of an exception than a norm. 
leader who makes proposals on behalf of her copartisans. The legislative session begins with a procedural stage in which the majority leader may propose to change the rule determining whether the minority can offer an amendment, and any rule change is subjected to a majority vote. I call the minority's ability to offer an amendment the minority right. Subsequent normal business of the legislature operates under a majority-rule amendment agenda in which the majority leader makes a policy proposal, and the minority leader offers an amendment only if the current rule allows for the possibility. This process repeats, and elections exogenously change the majority party and the placement of the legislative median over time.

In this framework, I uncover two results that link procedural persistence to the creation and endurance of minority rights. The main result is that when rules persist and the majority fears becoming the minority, legislators adopt and subsequently maintain the minority right to moderate future policy outcomes. This occurs even with ideologically polarized parties, some sessions that have unified majorities, and policymotivated legislators who do not use punishment strategies. The second result demonstrates that persistent rules are necessary for the right to emerge and substantively influence policy in this bargaining setting. More specifically, when the minority right is adopted in an equilibrium and procedures do not persist, another equilibrium exists with identical policy outcomes in which the right is never adopted. Likewise, persistent rules still encourage the legislature to adopt minority rights even in a more complicated environment with persistent policies.

These results emerge from the interaction between persistent rules and uncertainty over the future partisan composition of the legislature. When the partisan composition of the legislature potentially changes overtime, legislators in the majority fear becoming the minority in future sessions because they lose their agenda-setting power and the policy influence it entails. To preserve some of this influence, they institute the minority right, which allows them to subsequently moderate policy in future sessions where they become the minority. These rights survive over time because moderate, decisive legislators prevent leaders from removing rules that moderate policy. The possibility that the legislative median may be more moderate than the majority leader poses a strategic trade-off. On the one hand, the likelihood of joining the minority incentivizes majority leaders to introduce the right. On the other hand, 
the likelihood of remaining in the majority, but with a more moderate legislative median, encourages majority leaders to dissolve right because it dilutes her future agenda-setting powers. When the former likelihood is relatively large compared to the latter, the legislature adopts and subsequentlys maintain the minority right.

This mechanism connects several empirical regularities and accounts in the legislative organization literature. In the model, the minority right moves policy outcomes away from extreme leaders, and moderate legislators therefore defend the right, a trend found in the congressional politics literature (Schickler, 2000). Likewise, it is necessary for rules to persist for the minority right to emerge and actually moderate policy. When the majority must actively implement the right in every session, the minority does not substantively influence the equilibrium policy outcome even if the right is present, and a procedural advantage becomes a policy advantage as in cartel models of legislatures (Cox and McCubbins, 1993, 2005). Finally, the model highlights persistent rules as an important determinant of the variation in majority party agendasetting powers across legislative bodies, for example, why the Senate employs more inclusive policy-making rules than the House (Binder, 1997; Fenno, 1973; Smith, 2007). ${ }^{2}$ This analysis suggests that persistent rules encourage Senate majorities to preserve more open legislative institutions to moderate future policy outcomes, and these incentives are absent in the House, which is not a "continuing body."

The idea that the majority benefits from minority rights is common in the legislative institutions literature (e.g., Binder, 1996, 1997; Dion, 1997; Dixit et al., 2000; Koger, 2010; Wawro and Schickler, 2006). However, the current theory differs considerably from previous ones on two accounts. First, the majority party values the minority right to the extent that it potentially moderates future policies when it becomes the minority party. In my framework, legislators are policy motivated and do not gain informational benefits (Gilligan and Krehbiel, 1987; SukYoung Chwe, 1999; Wawro and Schickler, 2006), fear retaliation (Dixit et al., 2000; Koger, 2010), or value compromise with more moderate copartisans (Binder, 1997), which are other explanations for minority

\footnotetext{
${ }^{2}$ Although the two chambers employ different rules, debate exists over whether different rules translate into different levels of majority-party control (Campbell et al., 2002; Gailmard and Jenkins, 2007; Lazarus and Steigerwalt, 2009; Smith et al., 2013).
} 
rights. In other words, the theory here demonstrates that minority rights can still emerge and endure even with relatively sparse assumptions concerning legislators who, in the model, are policy motivated and do not possess private information.

Second, the theory does not require retaliation in the form of grimtrigger or tit-for-tat punishment strategies, and this is important for several reasons. Punishment strategies may be impractical in legislative settings (Krehbiel, 1991, p. 60). In addition, previous work has already demonstrated how history-dependent strategies generate political compromises in repeated games in the absence of persistent rules. (Alesina, 1988; Dixit et al., 2000; Fox, 2006). In contrast, for minority rights to be credible in my framework, there need exist only a possibility that moderate legislators are decisive in future sessions. This removes the need for punishment, and the next section illustrates the mechanism in a simpler finite setting where punishment is impossible. Furthermore, by restricting attention away from these types of strategies, the theory emphasizes the importance of rules to policy compromise. When legislators punish their colleagues for past actions, compromise does not require minority rights or any other legislative rules. Instead, future policy decisions themselves can be used to enforce cooperation with repeated interaction between patient parties. ${ }^{3}$ By focusing attention away from history-dependent strategies, the theory plausibly connects battles about minority rights to battles about policy.

Other efforts that endogenize proposal rights in a bargaining framework fix a closed or restrictive rule and allow legislators to allocate proposal prerogatives under this rule. In Yildirim (2007), legislators allocate proposal powers using a contest, and in Cotton (2012) a proposer retains her power if the proposal is successful or if she commands a majority. McKelvey and Riezman (1992) and Eguia and Shepsle (2013) investigate seniority rules, and Diermeier et al. (2013) examine conditions under which even moderate legislators restrict their personal proposal rights to decrease policy uncertainty. Jeon (2015) considers another form of persistence in the divide-the-dollar setting, where today's division determines tomorrow's proposal powers. In contrast to these previous works, the model below fixes proposal prerogatives within two

${ }^{3}$ To see this, note that the majority party solely determines policy in every period in the model from Dixit et al. (2000). 
policy-making procedures, i.e., open and closed agendas, but allows the legislature to adopt either rule. Most notably, Diermeier and Vlaicu (2011) also characterize conditions under which legislatures adopt open rather than closed rules, and they find that the majority grants the minority amendment rights only when members of the majority are just as likely to be on the same side of a random policy issue as members of the minority. In this sense, their result requires that parties have members with extremely diverse or uncorrelated preferences, which is not required for the results here.

Two important predecessors to this analysis are Krehbiel and Meirowitz (2002) and Krehbiel et al. (2015) who also study minority amendment rights. The former analyzes a two-dimensional policy space and finds that the power of the minority to affect policy depends on the location of the status-quo. The latter investigates a vote-buying framework and describes how the right moderates policy and encourages the majority leadership to buy votes. Both papers conclude with discussions about why minority rights emerge, and these echo some of the insight of the present analysis. Mainly, they conclude that "moderates (roughly, pivotal voters) tend to prefer the more open to the more closed procedure" (Krehbiel and Meirowitz, 2002, p. 212), and that "the majoriy recognizes that it will one day return to the minority" (Krehbiel et al., 2015, p. 19). Indeed, these incentives are essential to the main result of this paper but with an important caveat. Because the theory is explicitly dynamic, I find that minority rights are introduced and then supported even when the legislature begins with rules that favor the majority and there is no expectation of future punishment. In this case, a policy-motivated majority would have little incentive to first introduce the minority right without persistent rules.

\section{Illustration of the Mechanism}

To better understand the mechanism, consider a legislature comprising three actors: a party leader $A$, a rival leader $B$, and a median $\mu$, who is the representative, decisive legislator. Either leader $A$ or $B$ serves as the majority leader, and her rival serves as the minority leader. These actors are policy motivated with ideal points $\hat{x}_{A}=0, \hat{x}_{B}=1$ and $\hat{x}_{\mu}=\frac{1}{2}$, respectively, and I assume legislators have negative quadratic 
utilities. ${ }^{4}$ Suppose the normal business of the legislature (its policymaking process) operates using a majority-rule amendment agenda in which the majority leader proposes first. Subsequently, the minority leader offers an amendment if and only if the minority right is in place. When the status-quo policy is sufficiently bad, the adopted policy is the majority leader's ideal point without the right and the median's with the right. Essentially, with the minority right, two competing proposers drive policy to the median, and without the right, the majority leader capitalizes on her agenda-setting power, a standard result in RomerRosenthal bargaining with a bad status-quo. If the legislature lasts for one session only, neither leader has an incentive to introduce the right because it only moves policy away from their ideal points.

Now consider a legislature that lasts for two sessions and has persistent rules, that is, the procedure chosen in the first session becomes the default or status-quo procedure in the second. At the beginning of each session, legislators first decide whether the minority can offer amendments, and the rule changes only if both the majority leader and the median agree. Normal business remains the same, which means the legislature adopts the median's ideal point with the right and the majority leader's without the right. Even though rules persist, power does not. The majority leader in the first session retains her majority-status with probability $p$ and becomes the minority leader with probability $1-p$.

In this dynamic setting, the majority leader has an incentive to introduce the right. To see this, first note that the median always benefits under the minority right. This means that he not only accepts the right when it is proposed but also never allows it to be revoked when the right is currently in place. Thus, today's majority leader can tie the hands of tomorrow's leader by introducing the right. To see when this occurs, suppose $A$ is the majority leader in the first session and suppose the default rule precludes minority amendments. Figure 1 illustrates the two sequences of policies that arise from $A$ 's choice of procedures in the first session. If $A$ introduces the right, $\mu$ accepts and ensures the right is never revoked, leading to policy $\hat{x}_{\mu}$ in both sessions or a overall payoff of $-\frac{1}{2}$. If $A$ does not introduce the right,

\footnotetext{
${ }^{4}$ Negative quadratic utilities are important in this finite example, but less so in the infinitely repeated game. I discuss this in greater detail below.
} 


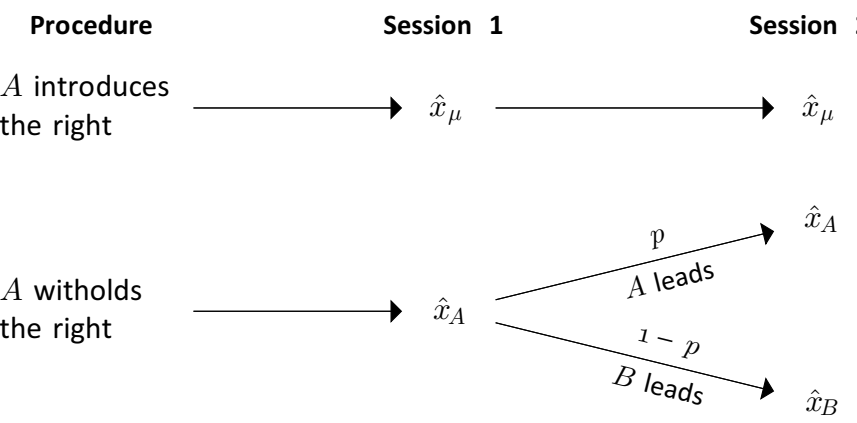

Figure 1: Policies in both sessions given rules today.

i.e., she keeps the closed procedure, she passes her ideal policy in the first session, but now there is uncertainty over future policies. With probability $p$, she retains her majority status, and her ideal policy is implemented. With probability $1-p$, she becomes the minority, and $B$ 's ideal policy is implemented. Thus, the legislative leadership introduces the right if and essentially only if $p<\frac{1}{2}$, and minority rights emerge even in this finite setting where punishment is impossible.

Although this example illustrates the dynamic incentive encouraging the legislature to adopt minority rights, it also reveals a large discrepancy between the majority leaders' strategies in the first and second sessions. Specifically, when $p$ is small, the majority leader always introduces the right in first session and never in the second. Furthermore, if leader $A$ knew that her rival would introduce the right in the second session, she would never do the same in the first even when $p$ is small. These discrepancies arise due to the finite nature of the interaction. An explicitly dynamic model is required to determine when these strategies emerge in a repeated setting. The model I construct addresses this issue, incorporates a more diverse institutional setting, and makes sharp predictions about when the legislature adopts and subsequently maintains minority rights.

\section{Model of Minority Rights}

In this section, I model a legislature that must first choose rules and then policies in each of an infinite number of sessions. As in the illustration above, the rules chosen in the current session become the default rules 
in the next session. Furthermore, the majority party does not know whether it will remain the majority and whether the party will become more or less moderate. To model this, consider the following dynamic game. Two political parties, Party $A$ and Party $B$, each contain two groups of legislators. A group is modeled as a single actor, an assumption which is addressed below. Specifically, Legislator 1 is the leader of Party $A$, and Legislator 4 is the leader of Party $B$. Parties $A$ and $B$ also include two moderate partisan Legislators 2 and 3, respectively. Label the set of legislators $N$.

All legislators $i$ have ideal policies $\hat{x}_{i}$ along the real line, which is also the policy space. The leaders' ideal policies are relatively more extreme than their copartisans' ${ }^{5}$ To model this, assume that Leaders 1 and 4 have ideal policies of 0 and 1 , respectively. The other two legislators' ideal policies are more moderate than their respective leaders by distance $d \in\left(0, \frac{1}{2}\right]$. That is, Legislator 2's ideal policy is $d$ and Legislator 3's is $1-d$. Hence, $d$ is a measure of intra-party or coalition heterogeneity, a characteristic of the current party system measuring preference diversity between coalition members. Figure 2 illustrates the four ideal points. Every legislator $i$ has instantaneous policy preferences represented by the quadratic utility function, $u_{i}(x)=-\left(x-\hat{x}_{i}\right)^{2}$ for all policies $x$.

Time is discrete and indexed by $t=1,2, \ldots, \infty$. Each period or session $t$ is initially characterized by two parameters or state variables. First, each period has a default legislative rule $\bar{r}_{t} \in\{\neg R, R\}$. Here $\bar{r}_{t}=R$ denotes the minority right under which the minority leader can

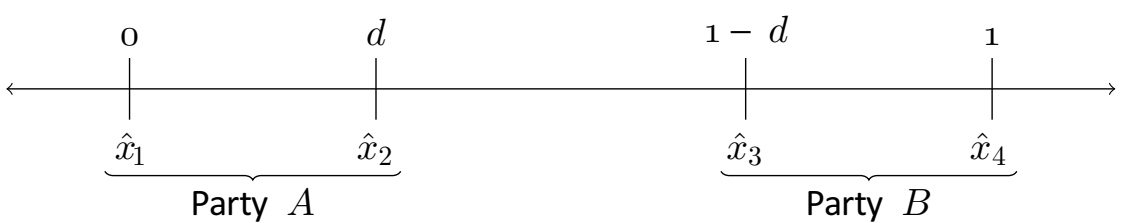

Figure 2: The layout of legislators' ideal points.

\footnotetext{
${ }^{5}$ It is also possible that there exist partisans groups more extreme than the leadership, but they are of no strategic consequence in this model and are omitted. In addition, Clausen and Wilcox (1987), Grofman et al. (2002), and King and Zeckhauser (2002) note that the majority leadership appears to be more extreme than than their average copartisan in the American context.
} 
offer an amendment in the proceeding policy stage, and $\bar{r}_{t}=\neg R$ is the absence of the minority right or majority dominance that precludes such an action. If the rule remains unchanged, it governs policy making in the period. Second, the composition of the legislature determines the median $\mu_{t}$, which must be one of the four legislators. Here, the median is the decisive legislator of the session, and also determines the majority/minority parties and leaders. Specifically, if the median is from Party $A$, Party $A$ is the majority party, $B$ is the minority party, and 1 and 4 are their respective leaders. The reverse holds when the median is from Party $B$. In other words, the partisanship of the median co-varies with the partisanship of the majority leader. Label the period's majority leader $M_{t}$ and the minority leader $m_{t}$.

The interaction in period $t$ proceeds in two stages. In the procedural stage, the majority leader $M_{t}$ proposes a rule $r_{t}^{p} \in\{\neg R, R\}$. The median legislator $\mu_{t}$ then chooses between the default rule and the proposed rule, and the choice becomes the procedural outcome $r_{t}$, governing the upcoming policy stage.

In the policy stage, the majority leader $M_{t}$ proposes a policy $x_{t}^{p} \in \mathbb{R}$, and the rule $r_{t}$ determines whether the minority can offer an amendment. With a minority right $\left(r_{t}=R\right)$, the minority leader $m_{t}$ proposes a competing policy amendment $x_{t}^{a} \in \mathbb{R}$, and the median $\mu_{t}$ chooses the majority's proposal, $x_{t}^{p}$, the minority's proposal, $x_{t}^{a}$, or some status quo $\bar{x}$ as the policy outcome. ${ }^{6}$ Under majority dominance $\left(r_{t}=\neg R\right)$, the median chooses $x_{t}^{p}$ or $\bar{x}$ as the policy outcome. In the baseline model, I assume the status-quo is some mutually disliked policy outside the interval $[0,1]$ although it is equivalent to assume that $\bar{x}$ is some different outcome that arises when no policy is passed. ${ }^{7}$ For example, if the policy space $[0,1]$ represents a division in the budget between guns and butter, $\bar{x}$ is the (very) bad outcome that materializes when no budget is passed. While this assumption is common in the legislative bargaining literature (e.g., cases when legislation pertains to appropriations with zero spending as a default), it may seem restrictive, so I later endogenize the status-quo policy in an extension.

\footnotetext{
${ }^{6}$ In this context, a simultaneous choice by the median is equivalent to a sequential agenda under majority rule with an odd number of voters.

${ }^{7}$ Formally, I require $u_{i}(x)>u_{i}(\bar{x})$ for all policies $x \in[0,1]$ and all legislators $i=1, \ldots, 4$, which holds when $\bar{x}$ is some real number outside $[0,1]$.
} 
After the end of the policy stage, the legislature transitions to the next session, and the two state variables governing policy-making change as well. First, rules persist, so the current rule becomes the default rule in the next session, that is, $\bar{r}_{t+1}=r_{t}$ for all periods $t$. In other words, today's rule remains in place until the majority leader and the median agree to change it. This makes the model consistent with the interpretation that the legislature is a "continuing body" where rules carry over from one session to the next as in the Senate.

Second, elections potentially change the composition of the legislature in the following manner. The majority party in period $t+1$ is the current majority party with probability $p \in(0,1)$. In words, $p$ represents the stickiness of power, i.e., the likelihood of the majority party and its leader retaining their positions between sessions. I hereafter refer to $p$ as the probability of retaining a majority. Regardless of the realization of the majority party, the majority leader is the legislative median with probability $q$, and say the majority is unified. With probability $1-q$, the majority leader is not the median, and say the majority is divided. Thus, a unified or divided majority coalition describes the current state of the legislature, regardless of the level of party heterogeneity $d$, which measures preference diversity within parties. Rather, the probability of a unified party represents a measure of party strength, and I refer to $q$ as party strength hereafter. For example, if $q$ is large, party leaders recruit or pressure their members to share their political goals and ideology, allowing them to maintain their leadership and median status.

Finally, actors' total payoffs are the discounted sum of their instantaneous utilities over policies with a common discount factor $\delta \in(0,1)$. That is, for some sequence of policy outcomes $\left\{x_{t}\right\}_{t=1}^{\infty}$, legislator $i$ 's total payoff is

$$
\sum_{t=1}^{\infty} \delta^{t-1} u_{i}\left(x_{t}\right),
$$

which completes the specification of the game.

Note that I have reduced a potentially very large legislature to one with four relevant actors. Because (weakly dominant) voting behavior with quadratic utilities and a common discount factor satisfy a sorting condition (Banks and Duggan, 2006), there is no strategic consequence to omitting non-median legislators who cannot propose. While this modeling decision pushes the leadership's proposal monopoly to the 


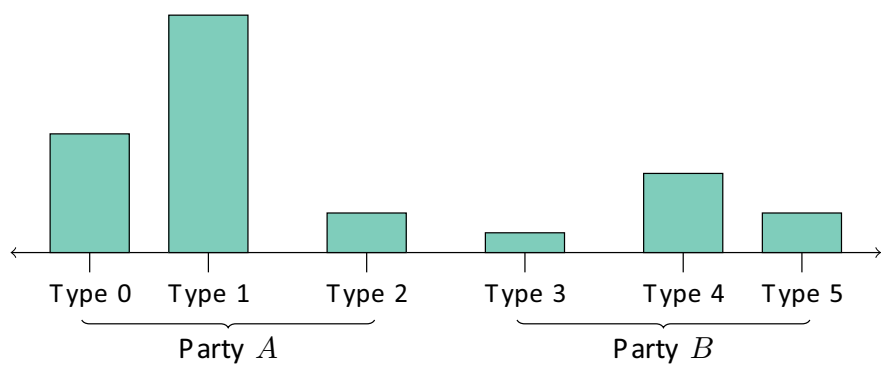

(a) Party $A$ is a unified majority, where $M_{t}=1$ and $\mu_{t}=1$.

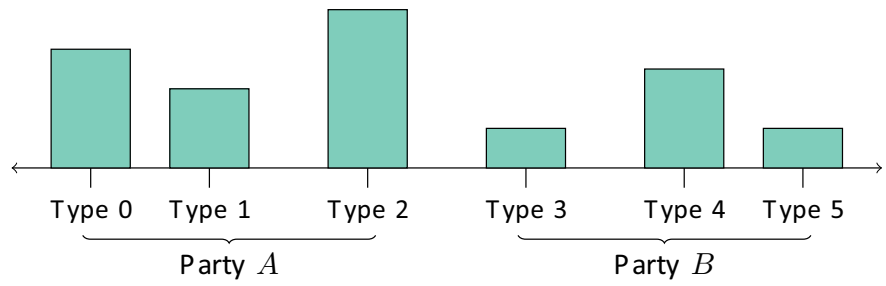

(b) Party $A$ is a divided majority, where $M_{t}=1$ and $\mu_{t}=2$.

Figure 3: Histograms of legislators' ideal points in two different states of the legislature.

extreme, I illustrate how the model changes when it is relaxed below. Figure 3 shows how the four legislators can represent a larger assembly by plotting the distribution of the legislative ideal points in two histograms with six potential types of legislators. In both histograms, Party $A$ is the majority party and a legislator from the type-1 group (Legislator 1) is the majority leader. Notice type- 1 legislators are relatively more extreme than the their more moderate, type- 2 copartisans but are less extreme than their type- 0 copartisans. (In fact, type 1 is the median of the majority party in these examples although this need not be the case.) However, in the first histogram, Figure 3(a), type-1 legislators are the median of the assembly, but in the second, Figure 3(b), type-2 legislators (legislator 2) are the median of the assembly. Legislators who have ideal points represented by types 0 and 5 only affect the decision-making process by determining the majority party and the legislative median. As such, these types of legislators, and potentially many others, need not be modeled. 


\section{Minority-Rights Equilibria}

In this section, I define the strategies, expected utilities, and equilibria. Throughout, I impose stationary and Markovian restrictions on the legislators' strategies in the sense that they can only condition on the payoff-relevant state variables. This is standard in these dynamics games and makes the analysis stronger as it does not rely on complicated punishment strategies to obtain equilibria which perpetuate the right. Accordingly, I drop references to the period $t$ below.

The previous section specified a game where within each period actors move sequentially. Let $g \in G$ denote each of the possible opportunities for actors to move within any period. I refer to $g$ as a decision node. More specifically, the set of decision nodes within a period is $G=\{r p, r c, p p, p a, m c, M c\}$, where $r p$ and $r c$ are the procedural proposal and choice nodes, and $p p$ and $a p$ the policy proposal and amendment nodes, and $m c$ and $M c$ the policy choices with and without the minority right. Figure 4 illustrates the interaction within a single period of the game and includes the labels of the decision nodes for reference.

At each decision node $g$, let $S^{g}$ denote the relevant state space and let $A^{g}$ denote the available actions. Throughout, I refer to states in $S^{r p}$ as initial states as they describe the initial characteristics of the legislative session. Table 1 describes the relevant state space and available action space at each decision node. For example, at the rule choice node $(g=r c)$, the relevant state space is the default rule $\bar{r}$, the legislative median $\mu$, and the majority leader's rule proposal $r^{p}$. Here, the legislative median can either accept the proposal $\left(1 \in A^{r p}\right)$ or not $\left(0 \in A^{r p}\right)$. Let $\sigma_{i}^{g}$ denote a function from $S^{g}$ to $A^{g}$, then a pure strategy for party leaders $i=1,4$ is a three-tuple $\sigma_{i}=\left(\sigma_{i}^{r p}, \sigma_{i}^{p p}, \sigma_{i}^{a p}\right){ }^{8}$ Likewise, a strategy for moderate legislators $i=2,3$ is also a three-tuple $\sigma_{i}=\left(\sigma_{i}^{r c}, \sigma_{i}^{m c}, \sigma_{i}^{M c}\right)$. Then, a strategy profile is four-tuple of strategies $\sigma=\left(\sigma_{1}, \ldots, \sigma_{4}\right)$, one for each legislator.

For every strategy $\sigma$ and every initial state $s^{r p} \in S^{r p}$ a unique policy and rule are implemented. Furthermore, this is true at every decision node. Let $x^{g}\left(s^{g} ; \sigma\right)$ denote the period's policy outcome following strategy

\footnotetext{
${ }^{8}$ Here, I implicitly assume that the majority leader chooses her policy proposal when she is the median. In addition, I allow the majority leaders to mix in the procedural stage in one result, and these strategies are discussed in the Appendix.
} 


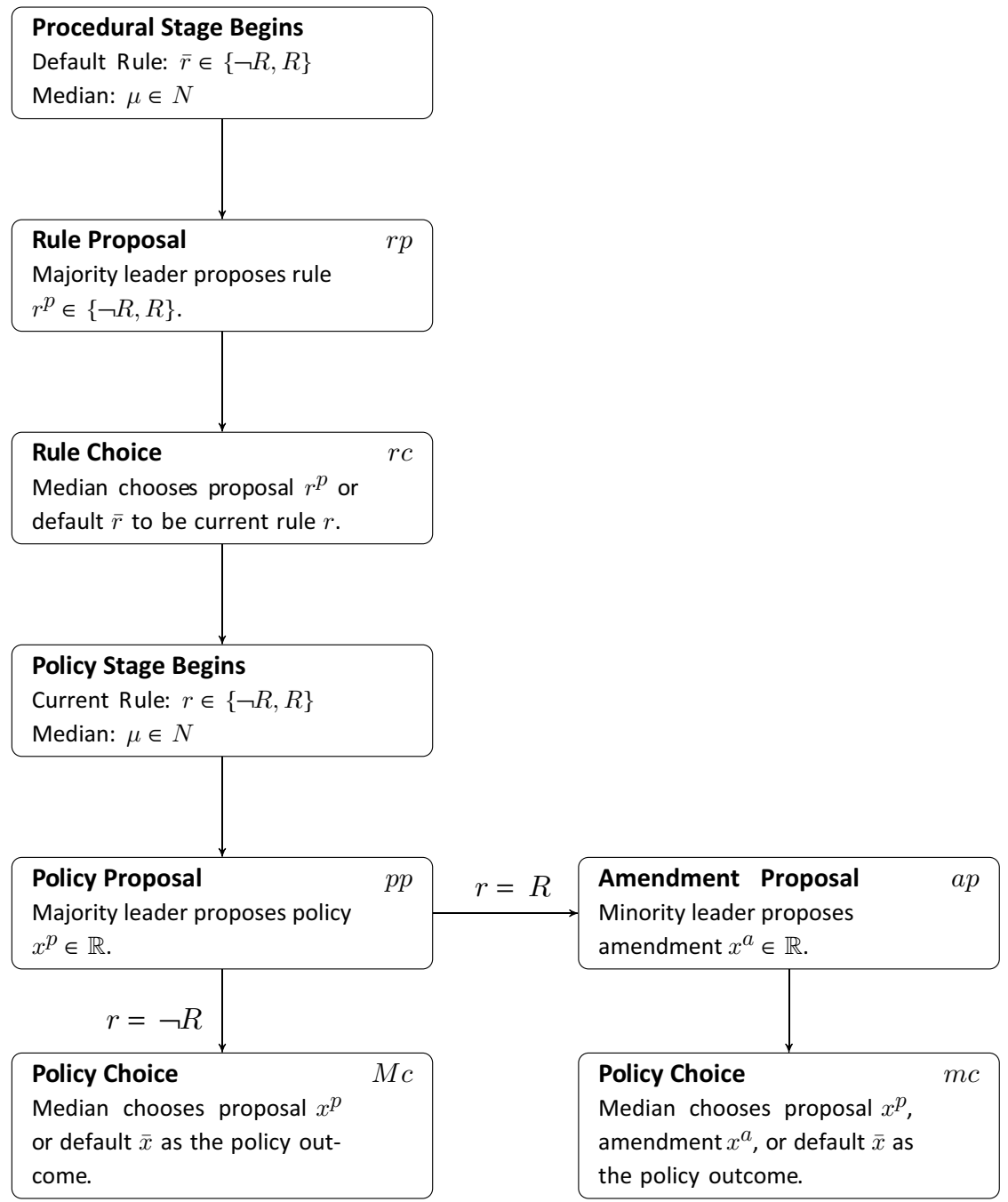

Figure 4: A diagram of the interaction within a period.

$\sigma$ from state $s^{g}$ at decision node $g$. In a similar manner, $r^{g}\left(s^{g} ; \sigma\right)$ is the procedural outcome following strategy $\sigma$ from state $s^{g}$. Let $\pi\left(\mu^{\prime} \mid \mu\right)$ denote the probability that legislator $\mu^{\prime}$ becomes the median in period $t+1$ given that the median is $\mu$ in period $t .^{9}$ This means legislator

\footnotetext{
${ }^{9}$ For example, if the current median is from Party $A$, then with probability $p q$ Legislator 1 is the median, with probability $p(1-q)$ Legislator 2 is the median, with
} 
Table 1: Description of state and action space at each decision node.

\begin{tabular}{lcc}
\hline Decision node $g$ & $\begin{array}{c}\text { Arbitrary } \\
\text { state } s^{g} \in S^{g}\end{array}$ & $\begin{array}{c}\text { Available } \\
\text { actions } A^{g}\end{array}$ \\
\hline Rule proposal $(r p)$ & $(\bar{r}, \mu)$ & $\{\neg R, R\}$ \\
Rule choice $(r c)$ & $\left(\bar{r}, \mu, r^{p}\right)$ & $\{0,1\}$ \\
Policy proposal $(p p)$ & $(r, \mu)$ & $\mathbb{R}$ \\
Amendment proposal $(a p)$ & $\left(\mu, x^{p}\right)$ & $\mathbb{R}$ \\
Policy choice with right $(m c)$ & $\left(\mu, x^{p}, x^{a}\right)$ & $\left\{c_{q}, c_{p}, c_{a}\right\}$ \\
Policy choice without right $(M c)$ & $\left(\mu, x^{p}\right)$ & $\left\{c_{q}, c_{p}\right\}$ \\
\hline
\end{tabular}

The relevant state $s^{g}$ is a vector summarizing the variables affecting an actors decision at node $g$. In the table, $\mu$ is the median legislator, $\bar{r}$ is a default rule in $\{R, \neg R\}, r^{p}$ is a rule proposal, and $x^{p}$ and $x^{a}$ are the policy and amendment proposals, which are from the real line. The right most column denotes the available actions at each decision node. Here, $c_{q}$ denotes the legislative median choosing the status-quo or default policy, $c_{p}$ the majority's proposal, and $c_{a}$ the minority's amendment.

$i$ 's dynamic expected utility of implementing policy $x$ and rule $r$ with median $\mu$ takes the form:

$U_{i}(x, r, \mu ; \sigma)=u_{i}(x)+\delta \sum_{\mu^{\prime} \in N} \pi\left(\mu^{\prime} \mid \mu\right) U_{i}\left(x^{r p}\left(r, \mu^{\prime} ; \sigma\right), r^{r p}\left(r, \mu^{\prime} ; \sigma\right), \mu^{\prime} ; \sigma\right)$.

Using Equation (1) it is straightforward to define a sub-game perfect equilibrium in stationary Markovian strategies (equilibrium hereafter), and the definition resides in the Appendix for reference.

I focus the analysis on equilibria that perpetuate minority rights, specifically those in which from any initial state and with probability one, the minority right is adopted and never removed. To define this, say $S \subseteq S^{r p}$ is an absorbing set with respect to profile $\sigma$ if for all $s=(\bar{r}, \mu) \in S$ and all $s^{\prime}=\left(\bar{r}^{\prime}, \mu^{\prime}\right) \in S^{r p}, r^{r p}(s ; \sigma)=\bar{r}^{\prime}$ implies $s^{\prime} \in S$. In other words, an absorbing set is a collection of initial states such that, for every state in the absorbing set, the procedural outcomes associated with the state transition to another state in the absorbing set. Because every legislator has a positive probability of becoming the next median regardless of the state, these transitions must include all

probability $(1-p)(1-q)$ Legislator 3 is the median, and with probability $(1-p) q$ Legislator 4 is the median. 
four medians. Say $S$ is an irreducible absorbing set with respect to $\sigma$ if there does not exist a strict subset $S^{\prime}$ of $S$ such that $S^{\prime}$ is an absorbing set with respect to $\sigma$. In other words, an irreducible absorbing set $S$ with respect to profile $\sigma$ contains all initial states such that once the path of play enters $S$, it never leaves the $S$ and every state in $S$ is reached with positive probability from some other state in $S$. My focus is on equilibria with irreducible absorbing sets that guarantee minority rights. The next definition states this formally.

Definition 1. An equilibrium $\sigma$ is a minority-rights equilibrium if all its of irreducible absorbing sets perpetuate the right, that is, for all irreducible absorbing sets $S$ with respect to $\sigma$ and all $s \in S, r^{r p}(s ; \sigma)=R$.

In other words, a minority-rights equilibrium ensures that if enough time passes the legislature adopts the right and perpetuates it in every proceeding period. This equilibrium concept is important for two reasons. First, a minority-rights equilibrium eventually perpetuates the right regardless of the initial state of the legislature. Thus, the path of play must enter an irreducible absorbing set in which legislators never remove the right. Second, in all other equilibria, there exists an initial state of the legislature such that if the path of play of begins in this state, the legislature never perpetuates the right indefinitely, i.e., the right vanishes along the path of play - potentially to reappear in future sessions. Thus, an analysis of minority-rights equilibria and their existence reveals when the legislature must perpetuate the right and when the right can be transitory.

\section{Results}

This section presents the main result of the paper: when rules persist and the probability of retaining a majority status is sufficiently small, the majority party first implements and then perpetuates the minority right. The result makes use of two preliminary lemmas, the intuition for which is straightforward, so formal proofs are omitted.

Lemma 1. In equilibrium, the following hold in every policy stage:

1. with majority dominance, the policy outcome is the majority leader's instantaneous ideal point; 
2. with the minority right, the policy outcome is the median's instantaneous ideal point;

3. with the minority right, the majority leader proposes the median's instantaneous ideal point.

The key to the intuition behind Lemma 1 is that current policies do not affect future payoffs because the default policy is constant in every state. Therefore, the policy outcome must be the majority leader's ideal point under majority dominance, as is standard in Romer-Rosenthal bargaining with a bad status-quo. Likewise, if the policy outcome is not the median's ideal point under the minority right, then either the majority or the minority leader has a profitable deviation by proposing such a policy, a result which does not depend on the status-quo policy due to the one-dimensional policy space. Now consider the sub-game in which the minority right is in place and the majority leader proposes a policy between her and the median's ideal points. Then the minority leader can extract some policy concessions from the median using her amendment powers. To prevent this, the majority leader proposes the median's ideal point under the open procedure in any equilibrium. Because the right drives policy to the median, the moderate Legislators 2 and 3 strictly prefer the minority right over majority dominance. The next result states this formally.

Lemma 2. Moderate medians strictly prefer the minority right in equilibrium.

In any equilibrium, policy outcomes must be the majority leader's ideal point under majority dominance and the median's under the minority right by Lemma 1 . Because the right only affects the policy payoffs under divided majorities, and in these cases the rule moves policy closer to the moderate median, the moderates can do no better than by instituting the right. They further receive strictly higher payoffs when they are themselves the median. ${ }^{10}$

Moderates' preference for open rules rather than closed ones is discussed in Binder (1997), Krehbiel and Meirowitz (2002), and Schickler

\footnotetext{
${ }^{10}$ Notice this result still holds when the status-quo, $\bar{x}$, resides in the unit interval as long $\bar{x}$ is not located at a median's ideal point.
} 
(2000), among others, and the two lemmas capture a similar logic. Furthermore, Lemma 2 demonstrates that when leaders must bargain with their more moderate copartisans over procedural changes, the moderates protect the right from an opportunistic leadership. Essentially, divided majorities never revoke the minority right, and this makes minority rights and their moderating benefits credible which drives the main result, stated as follows.

Proposition 1. There exist bounds $p^{+}$and $p^{*}$ on the probability of retaining a majority, $p$, such that $\frac{3}{4}<p^{+}<p^{*}<1$ and the following hold.

1. If $p<p^{*}$, a minority-rights equilibrium exists. If $p>p^{*}$, no equilibrium with an irreducible absorbing set that perpetuates the right exists.

2. Leaders proposing majority dominance in every procedural stage is an equilibrium if and only if $p \geq p^{+}$.

The Appendix contains the proof of this result and a detailed construction of minority-rights equilibria when $p<p^{*}$. In addition, Figure 5 provides an illustration of the main result. Most importantly, the proposition states that a minority-rights equilibrium exists if and essentially only if the probability of retaining a majority is not too large $\left(p<p^{*}\right)$. Furthermore, when $p<p^{+}$, the last result implies that, in every equilibrium, the right must be proposed in some initial state of the legislature. In contrast, when $p>p^{*}$, the legislature can never

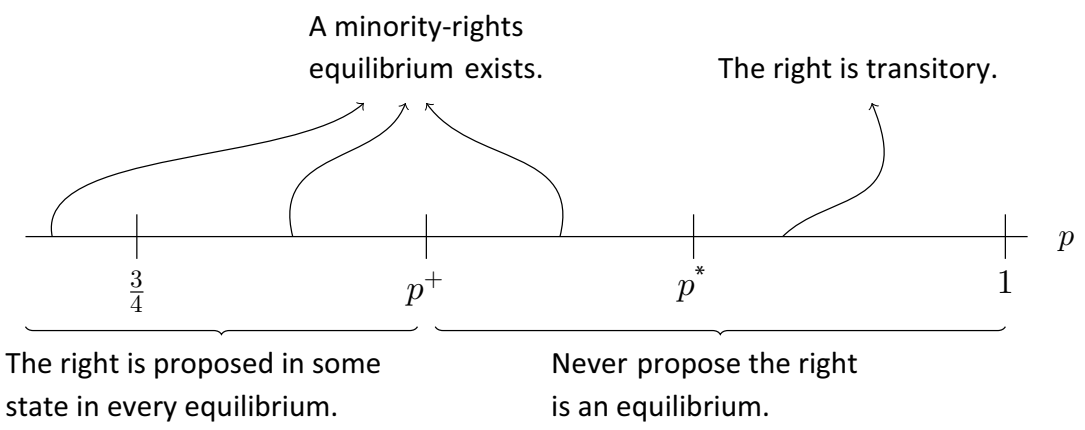

Figure 5: A summary of the main result in Proposition 1. 
perpetuate the right in any equilibrium, including a minority-rights equilibrium.

Thus, the model generates fairly sharp predictions about the existence and endurance of minority rights when the probability of retaining a majority is sufficiently large or small, i.e., $p<p^{+}$and $p>p^{*}$, but these predictions are less sharp when $p \in\left(p^{+}, p^{*}\right)$. However, even in this part of the parameter space there are reasons to suspect that legislators coordinate on a minority-rights equilibrium. When the majority is unified, all four legislators have larger dynamic utilities in a minorityrights equilibrium than in the equilibrium in which the right is never proposed. More generally, if the path of play were to get caught in an irreducible absorbing set in which the right is not perpetuated, Legislators 2 and 3 strictly prefer the stream of policies from a minority-rights equilibrium's unique irreducible absorbing set to the current one. This means that eventually a legislative majority prefers the absorbing set in minority-rights equilibria to those that do not perpetuate the right.

Notice the bounds $p^{+}$and $p^{*}$ are bounded below by $\frac{3}{4}$, which indicates that the majority leader must have a large retention probability before minority-rights equilibria cannot exist, and this specific bound is a direct result of the quadratic utilities. If, for example, stage utilities were $u_{i}=-\left|x-\hat{x}_{i}\right|^{\lambda}$, this bound is strictly increasing in $\lambda$, and $\lambda=1$ implies $p^{*}$ and $p^{+}$reduce to $\frac{1}{2} \cdot{ }^{11}$ In other words, while quadratic utilities are not necessary for the mechanism, sufficient risk aversion is required for safe majorities to institute the right.

The bounds $p^{+}$and $p^{*}$ are functions of party heterogeneity $d$, party strength $q$, and the patience of legislators, $\delta$. Party heterogeneity affects the bounds because the distance $d$ represents the benefits of instituting the right when a minority leader faces a divided majority and the cost when a majority leader oversees a divided majority. Party strength also affects the bounds because strong parties give the right little moderating benefits in future periods, thereby providing less incentive for the leadership to institute the right. Finally, as legislators become more patient, they internalize their chances of becoming the minority, regardless of how small the probability is. These relationships allow comparative statics on the bounds to illustrate the effect of the three

${ }^{11}$ Though such a set-up is potentially inconsistent with the representative-actor interpretation of the model, which requires quadratic utilities. 
parameters on the existence of a minority-rights equilibrium. The next proposition states these formally.

Proposition 2. 1. The bounds $p^{*}$ and $p^{+}$are increasing in legislators' patience, $\delta$, and decreasing in party heterogeneity, $d$. In addition $p^{+}$is decreasing in party strength, $q$, and $p^{*}$ is constant in $q$.

2. For every fixed $p$, if party heterogeneity is sufficiently small, then a minority-rights equilibrium exists, and in every equilibrium the minority right must be implemented in some initial states.

Although changes in patience, party strength, and party homogeneity all affect the likelihood that a minority-rights equilibrium exists, Proposition 2 reveals that party heterogeneity is particularly important. As party heterogeneity decreases, a minority-rights equilibrium is guaranteed to exist, and in every equilibrium a majority leader must propose the minority right. Intuitively, as heterogeneity $d$ approaches 0 , the majority leader's cost of implementing minority rights under a divided majority goes to zero, but, because legislators are risk averse, the expected benefits of implementing minority rights decrease at a slower rate. ${ }^{12}$ However, the other two parameters do not have such a substantial effect. For example, $p^{*}$ does not depend on $q$. In addition, as the legislators become perfectly patient, i.e., $\delta$ approaches 1 , $p^{*}$ converges to 1 , so a minority-rights equilibrium exits. Nonetheless, $p^{+}$remains bounded strictly below 1 , so there also exists an equilibrium in which the leaders never propose the right.

Before proceeding to the next section, I briefly comment on how the results in Proposition 1 remain robust to three changes in the model. First, it could be the case that the legislature uses a super-majority voting rule. To accommodate this possibility, I could incorporate two super-majority pivots for every possible median. Policy outcomes when the majority is unified would remain unchanged, and policy outcomes for divided majorities would be either the ideal point of the pivot closest to the majority leader or the leader's ideal point itself. This, however, is equivalent to reducing $d$ in the model. Furthermore, Proposition 2 then

${ }^{12}$ Obviously, when $d=0$, a minority-rights equilibrium and an equilibrium in which the right is never proposed exist because the right does not change current or future policy outcomes. 
suggests that super-majority rules themselves encourage minority rights because they have a similar effect to decreasing party heterogeneity.

Second, it could also be the case legislators who are more extreme than the leaders make policy proposals. More specifically, with probability $\pi$, the majority leader proposes in the policy stage as in the baseline model, but with probability $1-\pi$, a legislator more extreme makes the proposal. That is, when Legislator 1 (4) is the majority leader, a legislator with ideal point $\hat{x}_{0}<\hat{x}_{1}\left(\hat{x}_{5}>\hat{x}_{4}\right)$ proposes policy with probability $1-\pi$. Furthermore, assume that the result of this lottery over proposers is unknown to the majority leader during the procedural stage. Note that this extension does not affect policy outcomes when the right is in place but actually moves policy away from the leader's ideal with positive probability under majority dominance. This means that smaller values $\pi$ further encourage leaders to institute the right $\left(p^{*}\right.$ and $p^{+}$are increasing in $\pi$ ) because the possibility of extreme proposers decreases their agenda-setting benefits under majority dominance and the right still moderates policy when extremists propose.

Third, the main result also holds if the minority right were replaced with another procedure that allocates sole proposal power to the legislative median rather than the party leaders. In fact, the logic of my framework explains why majority leaders would delegate to or create proposal power for their more moderate copartisans. This suggests one reason why the legislative leadership does not fully monopolize proposal power even when such a monopoly is theoretically possible. Comparing these two types of procedures, i.e., assigning amendment power to the minority versus to moderate copartisans, demonstrates when minority rights substantively change policies: The moderate members of the majority party cannot construct bills or offer amendments. This speaks to a debate in the Congressional literature over whether the motion to recommit in the House affects policies (Krehbiel and Meirowitz, 2002; Roberts, 2005; Wolfensberger, 2007) and reflects a similar result in Roust (2005) who finds that motion-to-recommit amendments are never adopted along the path of play if open rules were previously used in the policy-making stage.

One reason why the two procedures in the preceding paragraph produce identical policy outcomes is the bad status-quo assumption in a one-dimensional policy space. This forces all proposers to have the same preference for policies between the two leader's ideal points over the 
default policy $\bar{x}$. When $\bar{x}$ consistently favors one party, say $\bar{x}$ resides in $\left(0, \hat{x}_{2}\right)$, for example, this encourages Leader 1 to introduce the minority right because her agenda-setting advantage under majority dominance is now diminished. Now, Leader 4 knows her opponent is more likely to introduce the right, reducing her incentives to do so, which suggests moderate default policies make minority-rights equilibria more difficult to sustain. Nonetheless, this relationship is an artifact of the asymmetry in the constant default policy. In the proceeding section, I address this issue by analyzing the model with persistent policies, a more realistic way to incorporate moderate status-quos.

\section{Necessity of Procedural Persistence}

In this section, I consider whether the mechanism underlying the previous results is robust to exogenous default rules and persistent policies. For example, the House cannot proceed to its policy-making capacity without first passing a set of rules, and this organizational structure may remove the possibility that rules carry over from session to session. Likewise, when policies persist, the policy enacted today becomes the status-quo or default policy tomorrow, and legislators do not always face a bad or constant status-quo as assumed in the previous section. The results in this section demonstrate necessity of procedural persistence for minority rights to appear. More specifically, when rules do not persist, the minority right cannot substantively affect policy outcomes, and when rules and policies persist, equilibria similar to those in the previous section exist in a reasonable set of parameters.

To investigate these versions of the model, I need the following notation and terminology. Let $\bar{x}_{t}$ denote the default or status-quo policy in period $t$. Now, initial states of the legislature take the form $s^{r p}=(\bar{x}, \bar{r}, \mu)$, and the remaining states require a similar modification. ${ }^{13}$ The first variation I consider is that the default rule in every procedural state is majority dominance but policies persist. ${ }^{14}$ Formally,

\footnotetext{
${ }^{13}$ These states and associated expected utility calculations are described in the Appendix.

${ }^{14}$ If the default rule is majority dominance and policies do not persist, the equilibrium is trivial: majority leaders never introduce the right when majority is divided, and they are indifferent between the right and majority dominance when the majority is unified.
} 
$\bar{r}_{t}=\neg R$ and $\bar{x}_{t+1}=x_{t}$ for all periods $t \geq 1 .{ }^{15}$ To see how the minority right affects policy outcomes with these transitions, first consider the definition of "policy equivalence" that is similar to the concept of payoff equivalence.

Definition 2. Two strategy profiles $\sigma$ and $\sigma^{\prime}$ are policy equivalent if, for all $s^{r p} \in S^{r p}, x^{r p}\left(s^{r p} ; \sigma\right)=x^{r p}\left(s^{r p} ; \sigma^{\prime}\right)$.

In words, the definition says that two strategy profiles are policy equivalent if, for all initial states, the policies produced under the two strategy profiles are the same regardless of the rules enacted. Because the policy outcomes associated with all states are the same, two policyequivalent profiles are payoff equivalent. Proposition 3 states the main result when rules do not persist.

Proposition 3. Assume that majority dominance is the default rule in every period and policies persist. For every equilibrium in which a majority leader proposes the minority right in some initial state of the legislature, there exists a policy-equivalent equilibrium such that the leader proposes majority dominance in the same state.

In other words, the minority does not substantively influence policies in equilibrium even if the right is present. Furthermore, the proposition immediately implies that for every minority-rights equilibrium, there exists a policy-equivalent equilibrium in which majority leaders never propose the right. The key to understand the result is that policy outcomes solely determine a legislator's expected utility in future periods regardless of the rule adopted in any procedural stage. Thus, when two equilibria are policy equivalent, they produce the same expected utilities for every legislator even though they may involve different rules. Because of this, the minority right cannot lead to better outcomes for the leadership when the default rule is majority dominance because, if it did, the majority leader could always enforce majority dominance and propose the other outcome.

Note that Proposition 3 demonstrates that minority rights do not substantively affect policy outcomes even when policies persist. To show that procedural persistence still encourages minority rights in this

\footnotetext{
${ }^{15}$ Note that policy persistence is the standard in legislative bargaining with an endogenous status-quo (e.g., Baron, 1996; Kalandrakis, 2004).
} 
more complicated setting, I also consider the case where both rules and policies persist, that is, $\bar{r}_{t+1}=\bar{r}_{t}$ and $\bar{x}_{t+1}=x_{t}$ for periods $t \geq 1$. Essentially, this variation relaxes the assumption of a bad status-quo in the baseline model. However, the analysis is complicated by the continuous nature of the default policy state variable. As is common in bargaining models, this leads to equilibria involving expected utility functions that are neither quasi-concave nor continuous in the policy outcome $x$ (Baron, 1996; Duggan and Kalandrakis, 2012; Kalandrakis, 2004; Zapal, 2011). Because of this, the proceeding result limits the parameter space under consideration, e.g., $p>\frac{1}{2}$ and $d$ is not too small, although no such restrictions on $\delta$ are necessary. In other words, the next proposition demonstrates that even with an electoral advantage, the majority leadership can still introduce and maintain the minority right.

Proposition 4. When rules and policies persist, there exists a minorityrights equilibrium in an open, non-empty set of parameters which does not depend on the discount factor, $\delta$, and requires that the probability of retaining a majority is sufficiently large, i.e., $p>1 / 2$. Furthermore, the equilibrium has the following properties:

- Leaders propose the right only when the majority is unified or the status-quo policy is sufficiently close to their respective moderate copartisan.

- Once the minority right is adopted, it is never revoked.

- Policy outcomes follow the median's ideal point when the legislature adopts the minority right.

- The majority leader engages in Romer-Rosenthal bargaining with the median before the minority right is adopted.

The specifics of the constructed equilibrium are in the Supplementary Information. In other words, Proposition 4 serves as a possibility result that the mechanism advanced in the previous sections is not affected when both rules and policies persist. Notice that the characterized equilibrium is quite similar to those in the previous section. However, there is an important modification to previous procedural strategies: both leaders propose the right when the majority is divided and the 
status quo is sufficiently close to their respective copartisan's ideal point. Essentially, moderate default policies also encourage a leader to propose minority rights with persistent rules and policies. This result contrasts with the discussion in the previous section, but the difference emerges because this extension does not force an asymmetric advantage in the default policy.

Taken together, these two results demonstrate the necessity of procedural persistence for minority rights to endure in legislatures and affect policy regardless of whether policies persist. More substantively, they suggest that even if formal minority rights exist in some legislatures, policies are immune from minority influence when rules do not persist. This relates to arguments in others studies on the House that connect the ability of the majority party to influence policies to its procedural dominance (Cox and McCubbins, 1993, 2005). Without procedural persistence, the right no longer affects the long-term calculations of the leaders as any majority leader can ensure her monopoly over the legislative agenda even in the presence of a moderate decisive legislator. More broadly, the stark contrast between Propositions 1 and 3 demonstrates the importance of procedural persistence when explaining inclusive policy-making processes and political compromises.

\section{Conclusion}

Legislative majorities often grant their minority counterparts significant rights to influence the policy-making process. One such right is the ability to amend bills subject to a majority vote. However, when legislators are purely policy motivated and cannot use punishment strategies, the emergence and endurance of these rights is not well understood in a bargaining framework, especially with cohesive and polarized parties or coalitions. Accounting for these considerations, I present a theory of minority rights that demonstrates why unified majorities perpetuate rules that grant the minority some agenda control. Unified majorities implement minority rights in order to moderate future policy outcomes when rules are persistent and there is uncertainty over their future majority status. These rights become permanent because moderate legislators benefit from competing policy proposals and defend these rights. Procedural persistence is a necessary requirement for 
minority rights to emerge in this framework. These results differ from previous accounts of endogenous proposal powers and demonstrate the importance of procedural persistence when explaining the development of inclusive political institutions.

In the bigger picture, the analysis here takes a first step toward explaining the emergence of amendment agendas and examining a legislative bargaining framework in which the decisive legislator changes over time. In addition, the theory also generates a rich set of comparative statics and empirical implications:

- As the probability of retaining a majority decreases, majority parties are more likely to introduce minority rights.

- If legislators' discount factors positively correlate with incumbency advantages or reelection rates, then larger incumbency advantages promote the adoption of minority rights.

- Weak parties, super-majority voting rules, extreme proposers, and moderate status-quo policies encourage minority rights.

In sum, the theory explains the endogenous creation and maintenance of legislative rules that moderate policy outcomes, which means the mechanism can easily be extended to explain minority rights such as resources to buy votes as in Krehbiel et al. (2015). Nonetheless, difficulties emerge in explaining rights that do not necessarily moderate policies such as obstructionism. Analyzing the emergence of this right requires characterizing, either by assumption or within an equilibrium, the distribution of status-quo policies. If these policies are close to the moderate legislators, then similar results would hold because obstructionism moderates policies. If these policies are extreme, moderate, decisive legislators may not always prefer the right, and therefore, members of the legislative majority face a more complicated strategic environment when creating a right to obstruct.

\section{Appendix}

\section{Expected Utilities and Equilibria}

I define $U_{i}^{g}\left(a, s^{g} ; \sigma\right)$ as $i$ 's expected utility of action $a$ at decision $g \in G$ given state $s^{g}$ and strategy profile $\sigma$. Recall that $x^{g}\left(s^{g} ; \sigma\right)$ and $r^{g}\left(s^{g} ; \sigma\right)$ 
denote the respective policy and procedural outcomes given decisionrelevant state $s^{g}$ and strategy profile $\sigma$. Below, I use $a$ to denote an arbitrary action from a discrete action set and $x$ to denote one from a continuous set.

We can write

$$
U_{i}^{M c}\left(a^{M c}, s^{M c} ; \sigma\right)=U_{i}^{m c}\left(a^{m c}, s^{m c} ; \sigma\right)= \begin{cases}U_{i}\left(x^{p}, r, \mu ; \sigma\right), & \text { if } a=c_{p} \\ U_{i}\left(x^{a}, r, \mu ; \sigma\right), & \text { if } a=c_{a} \\ U_{i}(\bar{x}, r, \mu ; \sigma), & \text { if } a=c_{q}\end{cases}
$$

for all $s^{M c} \in S^{M c}$, all $s^{m c} \in S^{m c}$, all $a^{M c} \in A^{M c}$, all $a^{m c} \in A^{m c}$ and all $i=2,3$. At the minority's amendment proposal, we write

$$
U_{i}^{a p}\left(x, s^{a p} ; \sigma\right)=U_{i}\left(x^{m c}\left(\mu, x^{p}, x ; \sigma\right), R, \mu ; \sigma\right)
$$

for all $x \in A^{a p}$, all $s^{a p} \in S^{a p}$, and all $i=1,4$. At the majority's proposal decision, we write

$$
U_{i}^{p p}\left(x, s^{a p} ; \sigma\right)=U_{i}\left(x^{a p}(r, \mu, x ; \sigma), r^{a p}(r, \mu, x ; \sigma), \mu ; \sigma\right)
$$

for all $x \in A^{p p}$, all $s^{a p} \in S^{a p}$, and all $i=1,4$. At the median's decision to accept a rule's proposal, we can write

$$
\begin{aligned}
U_{i}^{r c}\left(a, s^{r c} ; \sigma\right)= & U_{i}\left(x^{p p}\left(a r^{p}+(1-a) \bar{r}, \mu ; \sigma\right),\right. \\
& \left.\quad r^{p p}\left(a r^{p}+(1-a) \bar{r}, \mu ; \sigma\right), \mu ; \sigma\right) .
\end{aligned}
$$

for all $a \in A^{r c}=\{0,1\}$, all $s^{r c} \in S^{r c}$ and all $i=2,3$. At the majority's rule proposal, we write

$$
U_{i}^{r p}\left(a, s^{r p} ; \sigma\right)=U_{i}\left(x^{r c}(\bar{r}, \mu, a ; \sigma), r^{r c}(\bar{r}, \mu, a ; \sigma), \mu ; \sigma\right)
$$

for all $a \in\{\neg R, R\}$, all $s^{r p} \in S^{r p}$, and all $i=1,4$.

With this notation in hand, the next definition states the sub-game perfect equilibrium concept which precludes profitable deviations at each decision node.

Definition 3. A strategy profile $\sigma$ is an equilibrium if

$$
U_{i}^{g}\left(\sigma_{i}^{g}\left(s^{g}\right), s^{g} ; \sigma\right) \geq U_{i}^{g}\left(a, s^{g} ; \sigma\right)
$$

for all $g \in G$, all $s^{g} \in S^{g}$, all $a \in A^{g}$ and all $i \in N$. 
Proof of Proposition 3. Define the following:

$$
\begin{aligned}
& p^{+}=\frac{2-d(1+(1-q) \delta)}{2-2 d(1-q) \delta}, \\
& p^{-}=\frac{-d+(2-d)(1-q) \delta}{2(1-d)(1-q) \delta}
\end{aligned}
$$

and

$$
p^{*}=\frac{2-d(1+\delta)}{2-2 d \delta} \text {. }
$$

With some algebra, it can be shown that $\frac{3}{4}<p^{+}<p^{*}<1$ and the interval $\left[p^{-}, p^{+}\right]$is non-empty. To prove Proposition 1 , we first state an expanded version.

Proposition 1 (Expanded). There exist bounds $p^{-}, p^{+}$, and $p^{*}$ on the probability of retaining a majority, $p$, such that ${ }^{3} / 4<p^{+}<p^{*}<1$, $p^{-}<p^{+}$, and the following hold.

1. If $p<p^{*}$, a minority-rights equilibrium exists. If $p>p^{*}$, no equilibrium with an irreducible absorbing set that perpetuates the right exists.

2. Leaders proposing majority dominance in every procedural stage is an equilibrium if and only if $p \geq p^{+}$.

3. A minority-rights equilibrium in which only unified majorities propose the right exists if and only if $p^{-} \leq p \leq p^{+}$.

4. There exists a minority-rights equilibrium in mixed strategies when $p<p^{-}$in which unified majorities always propose the right and divided ones propose the right with probability strictly between 0 and 1.

5. There exists a minority-rights equilibrium in mixed strategies when $p \in\left(p^{+}, p^{*}\right)$ in which unified majorities propose the right with probability strictly between 0 and 1 and divided ones never propose the right. 
Proof of Proposition 1(Expanded)(1). Note that Proposition 1(Expanded)(3-5) demonstrates that a minority-rights equilibrium exists if $p<p^{*}$. We prove these below. For present purposes, it suffices to show that if $p>p^{*}$, then there does not exist an equilibrium $\sigma$ with an irreducible absorbing set $S$ that perpetuates the right, i.e., $r^{r g}(s ; \sigma)=R$ for all $s \in S$. To see this, suppose, contrary. Then there exists an irreducible absorbing set $\left\{\left(x_{0}, R, 1\right), \ldots,\left(x_{0}, R, 4\right)\right\}$, and the expected utility of these four states are given by the system of equations:

$$
\begin{aligned}
U_{1}(0, R, 1 ; \sigma)= & \delta\left(p q U_{1}(0, R, 1 ; \sigma)+p(1-q) U_{1}(d, R, 2 ; \sigma)\right. \\
& +(1-p) q U_{1}(1, R, 4 ; \sigma) \\
& \left.+(1-p)(1-q) U_{1}(1-d, R, 3 ; \sigma)\right), \\
U_{1}(d, R, 2 ; \sigma)= & -d^{2}+U_{1}(0, R, 1 ; \sigma), \\
U_{1}(1, R, 4 ; \sigma)= & -1+\delta\left(p q U_{1}(1, R, 4 ; \sigma)+p(1-q) U_{1}(1-d, R, 3 ; \sigma)\right. \\
& +(1-p) q U_{1}(0, R, 1 ; \sigma) \\
& \left.+(1-p)(1-q) U_{1}(1-d, R, 3 ; \sigma)\right),
\end{aligned}
$$

and

$$
U_{1}(1-d, R, 3 ; \sigma)=1-(1-d)^{2}+U_{1}(1, R, 4 ; \sigma) .
$$

Solving reveals,

$$
\begin{aligned}
& U_{1}(0, R, 1 ; \sigma)=-\frac{\delta\left(1-p-2 d(1-p)(1-q)+d^{2}(1-q)(1+(1-2 p) \delta)\right)}{(1-\delta)(1+(1-2 p) \delta)}, \\
& U_{1}(1, R, 4 ; \sigma)=\frac{-1+\delta(p-d(1-q)(d-2 p-(2-d)(1-2 p) \delta))}{(1-\delta)(1+(1-2 p) \delta)},
\end{aligned}
$$

and

$$
\begin{aligned}
& U_{1}(d, R, 2 ; \sigma) \\
& \quad=\frac{-d^{2}(1+(1-2 p) \delta)(1-q \delta)+2 d(1-p)(1-q) \delta-(1-p) \delta}{(1-\delta)(1+(1-2 p) \delta)} .
\end{aligned}
$$


Furthermore, it must be the case that

$$
\sigma_{i}^{r p}(R, i)=R
$$

for $i=1,4$ or else $\left\{\left(x_{0}, R, 1\right), \ldots,\left(x_{0}, R, 4\right)\right\}$ would not be an irreducible set. In addition, we require that there exist $s^{r p}=(\bar{r}, \mu) \in S^{r p}$ such that $\bar{r}=\neg R$ and either $\sigma_{1}^{r p}\left(s^{r p}\right)=R$ and $\mu \in\{1,2\}$ or $\sigma_{4}^{r p}\left(s^{r p}\right)=R$ and $\mu \in\{3,4\}$. Without loss of generality, suppose the former. Because $\sigma$ is an equilibrium, we require $U^{r p}\left(\neg R, s^{r p} ; \sigma\right) \leq U^{r p}\left(R, s^{r p} ; \sigma\right)$.

Now, Leader 1's expected utility of retaining majority dominance in a minority-rights equilibrium and in state $s^{r p} \in S^{r p}$ is minimized when $\sigma_{4}^{r p}\left(s^{r p^{\prime}}\right)=\neg R$ for all $s^{r p^{\prime}} \in S^{r p} \backslash\{(R, 4)\}$. (The state $(R, 4)$ needs to be excluded or else there would be no absorbing set in which only the minority is implemented.) So assume that is the case. Furthermore, Leader 1's expected utility of implementing the minority right is maximized when she does this with a unified party, i.e., $\mu=i$, and she proposes majority dominance with a divided party. Again, suppose this is the case. We now show that even under these most favorable conditions, Leader 1 can profitably deviate by not offering minority rights in state $(\neg R, 1)$, that is, we show $U^{r p}(\neg R,(\neg R, 1) ; \sigma)>U^{r p}(R,(\neg R, 1) ; \sigma)$.

To see this, we can consider the expected utility of Legislator 1 implementing $\neg R$ in the state $s^{r p}=(\neg R, 1)$ and then immediately return to a strategy in which she only proposes the minority right when unified. By Lemma 1, she would receive

$$
\begin{aligned}
U_{1}(0, \neg R, 1 ; \sigma)= & \delta\left(p q U_{1}(0, R, 1 ; \sigma)+p(1-q) U_{1}(0, \neg R, 1 ; \sigma)\right. \\
& \left.+(1-p) U_{1}(0, \neg R, j ; \sigma)\right),
\end{aligned}
$$

and

$$
\begin{aligned}
U_{1}(0, \neg R, j ; \sigma)= & -1+\delta\left(p U_{1}(0, \neg R, j ; \sigma)+(1-p) q U_{1}(0, R, 1 ; \sigma)\right. \\
& \left.+(1-p)(1-q) U_{1}(0, \neg R, 1 ; \sigma)\right),
\end{aligned}
$$

where $j \in\{3,4\}$. Solving these two equations give us

$$
U_{1}(0, \neg R, 1 ; \sigma)=\delta \frac{1-p\left(1+q U_{1}(0, R, 1 ; \sigma)\right)-(1-2 p) q \delta U_{1}(0, R, 1 ; \sigma)}{-1+p(2-q) \delta+(1-2 p)(1-q) \delta^{2}}
$$


For Leader 1 to not have a profitable deviation in state $s^{r p}=(R, 1)$, we require

$$
\begin{aligned}
& U_{1}(0, R, 1 ; \sigma)-U_{1}(0, \neg R, 1 ; \sigma) \geq 0 \\
& \Longleftrightarrow-\frac{d(1-q) \delta(2-2 p-d(1+(1-2 p) \delta))}{-1+p(2-q) \delta+(1-2 p)(1-q) \delta^{2}} \geq 0 \\
& \Longleftrightarrow p \leq \frac{2-d(1+\delta)}{2-2 d \delta}=p^{*},
\end{aligned}
$$

but this cannot be the case because $p>p^{*}$.

Proof of Proposition 1 (Expanded)(2). Consider a strategy profile $\sigma$ such that $\sigma_{i}^{r p}\left(s^{r p}\right)=\neg R$ for all $s^{r p} \in S^{r p}$. Suppose $\sigma$ is an equilibrium. We must verify that $U_{i}^{r p}\left(\neg R, s^{r p} ; \sigma\right) \geq U_{i}^{r p}\left(R, s^{r p} ; \sigma\right)$ for all $i=1,4$ and all $s^{r p} \in S^{r p}$. We only prove this for leader 1 as the condition for leader 4 is symmetric. Three observations help in the proof. First, if $\sigma$ is an equilibrium, the condition indeed holds for $s^{r p}$ in which $\bar{r}=R$ and $\mu=2$ because the procedural outcome will be $R$ regardless of leader 1's strategy. This follows from Lemma 2. Second, we need only verify the inequality in states such that $\mu=1$. If the condition holds in these states, it will also hold in states with median $\mu=2$ because $U_{1}^{r p}(R,(\neg R, 1) ; \sigma)>U_{1}^{r p}(R,(\neg R, 2) ; \sigma)$ when the leader must incur an immediate policy loss from implementing the minority right. Finally, these two comments and Lemma 1 imply that it suffices to show that $U_{1}(0, \neg R, 1 ; \sigma) \geq U_{1}(0, R, 1 ; \sigma)$.

We can write the expected utility of Leader 1 from following $\sigma$ in a state $s^{r p}$ such that $\mu=1$ and $\bar{r}=\neg R$ as

$$
\begin{aligned}
U_{1}(0, \neg R, 1 ; \sigma)= & \delta\left(p q U_{1}(0, \neg R, 1 ; \sigma)+p(1-q) U_{1}(0, \neg R, 2 ; \sigma)\right. \\
& +(1-p) q U_{1}(1, \neg R, 4 ; \sigma) \\
& \left.+(1-p)(1-q) U_{1}(1, \neg R, 3 ; \sigma)\right)
\end{aligned}
$$

and

$$
\begin{aligned}
U_{1}(1, \neg R, 4 ; \sigma)= & -1+\delta\left(p q U_{1}(1, \neg R, 4 ; \sigma)+p(1-q) U_{1}(1, \neg R, 3 ; \sigma)\right. \\
& +(1-p) q U_{1}(0, \neg R, 1 ; \sigma) \\
& \left.+(1-p)(1-q) U_{1}(0, \neg R, 2 ; \sigma)\right) .
\end{aligned}
$$


Because the immediate policy payoffs and transition probabilities are identical, $U_{1}(0, \neg R, 1 ; \sigma)=U_{1}(0, \neg R, 2 ; \sigma)$ and $U_{1}(1, \neg R, 4 ; \sigma)=$ $U_{1}(1, \neg R, 3 ; \sigma)$. Solving reveals

$$
U_{1}(0, \neg R, 1 ; \sigma)=\frac{-\delta(1-p)}{(1-\delta)(1+(1-2 p) \delta)}
$$

and

$$
U_{1}(1, \neg R, 4 ; \sigma)=\frac{-1+p \delta}{(1-\delta)(1+(1-2 p) \delta)}
$$

Now suppose Leader 1 were to deviate by offering $R$ and immediately return to playing $\sigma$ when she is the median, i.e., $\mu=1$. The expected utility of the deviation is

$$
\begin{aligned}
U_{1}(0, R, 1 ; \sigma)= & \delta\left(p q U_{1}(0, \neg R, 1 ; \sigma)+p(1-q) U_{1}(d, R, 2 ; \sigma)\right. \\
& +(1-p) q U_{1}(1, \neg R, 4 ; \sigma) \\
& \left.+(1-p)(1-q) U_{1}(1-d, R, 3 ; \sigma)\right)
\end{aligned}
$$

where

$$
U_{1}(d, R, 2 ; \sigma)=-d^{2}+U_{1}(0, R, 1 ; \sigma)
$$

and

$$
\begin{aligned}
U_{1}(1-d, R, 3 ; \sigma)= & -(1-d)^{2}+\delta\left(p q U_{1}(1, \neg R, 4 ; \sigma)\right. \\
& +p(1-q) U_{1}(1-d, R, 3 ; \sigma) \\
& +(1-p) q U_{1}(0, \neg R, 1 ; \sigma) \\
& \left.+(1-p)(1-q) U_{1}(d, R, 2 ; \sigma)\right) .
\end{aligned}
$$

Solving gives us

$$
\begin{aligned}
U_{1}(0, R, 1 ; \sigma)= & \frac{-(1-q) \delta(1-p-d(2-2 p-d(1+(1-2 p)(1-q) \delta)))}{1-(1-q) \delta(2 p+(1-2 p)(1-q) \delta)} \\
& +\delta U_{1}(0, \neg R, 1 ; \sigma) \frac{q(p+(1-2 p)(1-q) \delta)}{1-(1-q) \delta(2 p+(1-2 p)(1-q) \delta)} \\
& +\delta U_{1}(1, \neg R, 4 ; \sigma) \frac{q(1-p)}{1-(1-q) \delta(2 p+(1-2 p)(1-q) \delta)}
\end{aligned}
$$


We require

$$
\begin{aligned}
U_{1}(0, & \neg R, 1 ; \sigma)-U_{1}(0, R, 1 ; \sigma) \geq 0 \\
& \Longleftrightarrow d\left(-d+\frac{d-1}{1-(1-q) \delta}+\frac{1}{1+(1-2 p)(1-q) \delta}\right) \geq 0 \\
& \Longleftrightarrow p \geq \frac{2-d(1+(1-q) \delta)}{2+2 d(1-q) \delta}=p^{+} .
\end{aligned}
$$

Existence is by construction. In the policy stage with minority right, Legislators 2 and 3 choose the policy that maximizes their instantaneous utilities $u_{2}$ and $u_{3}$, but they defer to the minority leader when indifferent between the two leaders' proposals. Specifically, for $i=2,3$

$$
\sigma_{i}^{M c}\left(s^{M c}\right)=\sigma_{i}^{m c}\left(s^{m c}\right)= \begin{cases}c_{a}, & \text { if } x^{a} \in \arg \max _{x^{\prime} \in\left\{\bar{x}, x^{p}, x^{a}\right\}} u_{i}\left(x^{\prime}\right) \\ c_{p}, & \text { if } x^{p} \in \arg \max _{x^{\prime} \in\left\{\bar{x}, x^{p}, x^{a}\right\}} u_{i}\left(x^{\prime}\right) \\ & \text { and } x^{a} \notin \arg \max _{x^{\prime} \in\left\{\bar{x}, x^{p}, x^{a}\right\}} u_{i}\left(x^{\prime}\right) \\ c_{q}, & \text { otherwise. }\end{cases}
$$

The minority leader proposes a policy that maximizes her instantaneous payoffs subject to the majority leader's proposal. For example, we can write

$$
\hat{\sigma}_{1}^{a p}\left(s^{a p}\right)= \begin{cases}0, & \text { if }\left|\hat{x}_{1}-\hat{x}_{\mu}\right| \leq\left|x^{p}-\hat{x}_{\mu}\right| \\ \mu-\left|x^{p}-\hat{x}_{\mu}\right|, & \text { otherwise }\end{cases}
$$

for 1 when she is the minority leader, and

$$
\hat{\sigma}_{4}^{a p}\left(s^{a p}\right)= \begin{cases}1, & \text { if }\left|\hat{x}_{4}-\hat{x}_{\mu}\right| \leq\left|x^{p}-\hat{x}_{\mu}\right| \\ \mu+\left|x^{p}-\hat{x}_{\mu}\right|, & \text { otherwise }\end{cases}
$$

for Leader 4. Under a policy stage with the right, the majority leader should always propose the median's ideal policy. That is, $\sigma_{i}^{p p}\left(s^{p p}\right)=\hat{x}_{\mu}$ for leaders $i=1,4$ and $s^{p p} \in S^{p p}$ such that $r=R$. So, there is no profitable deviation in a policy stage with the right. Under majority dominance, the majority leader proposes her ideal point, i.e., $\sigma_{i}^{p p}\left(s^{p p}\right)=$ $\hat{x}_{i}$ for $i=1,4$ and $s^{p p} \in S^{p p}$ such that $r=\neg R$. This is identical to a repeated Romer-Rosenthal bargaining game, so there is no profitable deviation. In the procedural stage, we need the moderate medians $i=2,3$ to accept a procedural proposal if and only if the proposal 
is the minority right. Finally, the leaders should never propose the right. When the default rule is $R$ and the majority is divided, the leader cannot change the proceeding policy outcome regardless of her proposal. When the default rule is $\neg R$, we only need to rule out profitable deviations when the leaders are the median, because this will also rule out profitable deviations when they control a divided majority. By the previous discussion, this means we need to verify $U_{1}(0, \neg R, 1 ; \sigma) \geq U_{1}(0, R, 1 ; \sigma)$, but this inequality holds precisely when $p \geq p^{+}$.

Proof of Proposition 1(Expanded)(3). Consider a strategy profile $\sigma$ such that $\sigma_{i}^{r p}\left(s^{r p}\right)=R$ if $\mu=i$ and $\sigma_{i}^{r p}\left(s^{r p}\right)=\neg R$ otherwise for leaders $i=1,4$ Suppose $\sigma$ is an equilibrium and $p \in\left[p^{-}, p^{+}\right]$. We must verify that for $i=1,4, U_{i}^{r p}\left(R, s^{r p} ; \sigma\right) \geq U_{i}^{r p}\left(\neg R, s^{r p} ; \sigma\right)$ for all $s^{r p} \in S^{r p}$ such that $\mu=i$ and $U_{i}^{r p}\left(\neg R, s^{r p} ; \sigma\right) \geq U_{i}^{r p}\left(R, s^{r p} ; \sigma\right)$ for all $s^{r p} \in S^{r p}$ such that $\mu \neq i$.

Note that $U_{i}^{r p}\left(r^{p}, s^{r p} ; \sigma\right)=U_{i}^{r p}\left(r^{p}, s^{r p^{\prime}} ; \sigma\right)$ for two state $s^{r p}$ and $s^{r p^{\prime}}$ such that $i=\mu=\mu^{\prime}$ because the leader's rule choice will always be implemented. Further when the state $s^{r p}$ is such that $\bar{r}=R$ and $\mu \neq i$, the rule proposal will not have an effect on the subsequent policy outcome because right will remain in effect. Using Lemmas 1 and 2 and symmetry, it now suffices to show $U_{1}(0, R, 1 ; \sigma) \geq U_{1}(0, \neg R, 1 ; \sigma)$ and $U_{1}(0, \neg R, 2 ; \sigma) \geq U_{1}(d, R, 2 ; \sigma)$. The values $U_{1}(0, R, 1 ; \sigma)$ and $U_{1}(d, R, 2 ; \sigma)$ are those that solve Equations (8)-(10).

First we verify $U_{1}(0, R, 1 ; \sigma) \geq U_{1}(0, \neg R, 1 ; \sigma)$. Suppose Leader 1 deviates by enforcing $\neg R$ in a procedural period in which she is the median and then immediately returns to strategy $\sigma$. The outcome of the policy stage would be 0 , and majority dominance would remain in place as long as the majority party remains divided. Therefore, we can compute the expected utility of her deviation as follows. Write

$$
\begin{aligned}
U_{1}(0, \neg R, 1 ; \sigma)= & \delta\left(p q U_{1}(0, R, 1 ; \sigma)+p(1-q) U_{1}(0, \neg R, 2 ; \sigma)\right. \\
& +(1-p) q U_{1}(1, R, 4 ; \sigma) \\
& \left.+(1-p)(1-q) U_{1}(1, \neg R, 3 ; \sigma)\right),
\end{aligned}
$$

where

$$
\begin{aligned}
U_{1}(1, \neg R, 3 ; \sigma)= & -1+\delta\left(p q U_{1}(1, R, 4 ; \sigma)+p(1-q) U_{1}(1, \neg R, 3 ; \sigma)\right. \\
& +(1-p) q U_{1}(0, R, 1 ; \sigma) \\
& \left.+(1-p)(1-q) U_{1}(0, \neg R, 2 ; \sigma)\right) .
\end{aligned}
$$


Note that $U_{1}(0, \neg R, 1 ; \sigma)=U_{1}(0, R, 2 ; \sigma)$ because the median only influences transition probabilities, which vary by majority/minority status. Solving, we can write

$$
\begin{gathered}
\delta(1-p)\left(-1+q+U_{1}(1, R, 4 ; \sigma)\right) \\
U_{1}(0, \neg R, 1 ; \sigma)=\frac{+\delta q U_{1}(0, R, 1 ; \sigma)(p+(1-2 p)(1-q) \delta)}{1-(1-q) \delta(2 p+(1-2 p)(1-q) \delta)} .
\end{gathered}
$$

Because $\sigma$ is an equilibrium, we require

$$
\begin{aligned}
& U_{1}(0, R, 1 ; \sigma)-U_{1}(0, \neg R, 1 ; \sigma) \geq 0 \\
& \quad \Longleftrightarrow d\left(-d+\frac{d-1}{1-(1-q) \delta}+\frac{1}{1+(1-2 p)(1-q) \delta}\right) \geq 0 \\
& \quad \Longleftrightarrow p \leq \frac{2-d(1+(1-q) \delta)}{2+2 d(1-q) \delta}=p^{+},
\end{aligned}
$$

as required.

Second, we characterize the conditions under which $U_{1}(0, \neg R, 2 ; \sigma) \geq$ $U_{1}(d, R, 2 ; \sigma)$. To compute $U_{1}(0, \neg R, 2 ; \sigma)$, we write

$$
\begin{aligned}
U_{1}(0, \neg R, 2 ; \sigma)= & \delta\left(p q U_{1}(0, R, 1 ; \sigma)+p(1-q) U_{1}(0, \neg R, 2 ; \sigma)\right. \\
& \left.+(1-p) q U_{1}(1, R, 4 ; \sigma)+(1-p)(1-q) U_{1}(1, \neg R, 3 ; \sigma)\right) \\
= & U_{1}(0, \neg R, 1 ; \sigma) .
\end{aligned}
$$

So we require

$$
\begin{aligned}
U_{1}(0, & \neg R, 1 ; \sigma)-U_{1}(d, R, 2 ; \sigma) \geq 0 \\
& \Longleftrightarrow \frac{-1+d}{1-(1-q) \delta}+\frac{1}{1+(1-2 p)(1-q) \delta} \geq 0 \\
& \Longleftrightarrow p \geq \frac{-d+(2-d)(1-q) \delta}{2(1-d)(1-q) \delta}=p^{-}
\end{aligned}
$$

Existence follows from construction, and the construction is similar to the one in the previous proof.

Proof of Proposition 1(Expanded)(4). I first introduce mixed strategies in the procedural stage and show how to calculate expected utilities 
under mixed strategies. A mixed rule proposal strategy for legislator $i=1,4$ is a function $\gamma_{i}^{r p}: S^{r p} \rightarrow[0,1]$ such that $\gamma_{i}^{r p}\left(s^{r p}\right)$ denotes the probability with which leader $i$ proposes the minority right. In a slight abuse of notation, a strategy for $i=1,4$ becomes a three-tuple $\sigma_{i}=\left(\gamma_{i}^{r p}, \sigma_{i}^{p p}, \sigma_{i}^{a p}\right)$. The strategy for $i=2,3$ remains unchanged.

Given strategy profile $\sigma$ and some initial state $s^{r p}$, there exists a probability function $\tilde{\pi}$ over potential procedural outcomes and policy outcomes, where $\tilde{\pi}\left(x, r \mid s^{r p} ; \sigma\right)$ denotes the probability that policy $x$ and rule $r$ are implemented give state $s^{r p}$, and strategy profile $\sigma$. This is a probability function because there are a finite number of rules and actors employ pure strategies in the policy stage. Let $\mathrm{S}_{\sigma}^{\tilde{\pi}}\left(s^{r p}\right)$ denote the support of $\tilde{\pi}$ with initial state $s^{r p}$ and strategy profile $\sigma$. We can write expected utility calculations as follows

$$
\begin{aligned}
U_{i}(x, r, \mu ; \sigma)= & u_{i}(x)+\delta \sum_{\mu^{\prime} \in N} \pi\left(\mu^{\prime} \mid \mu\right) \\
& \times \sum_{\substack{\left(x^{\prime}, r^{\prime}\right) \in \\
\mathrm{S}_{\sigma}^{\tilde{\pi}}\left(r, \mu^{\prime}\right)}} \tilde{\pi}\left(x^{\prime}, r^{\prime} \mid\left(r, \mu^{\prime}\right) ; \sigma\right) U_{i}\left(x^{\prime}, r^{\prime}, \mu^{\prime} ; \sigma\right) .
\end{aligned}
$$

For a strategy profile $\sigma$ to be an equilibrium, we require the condition in Definition 3 for all pure strategies. However, under mixed strategies, we require that in any state $s^{r p}$

$$
\gamma_{i}^{r p}\left(s^{r p}\right) \in(0,1) \Longrightarrow U_{i}^{r p}\left(R, s^{r p} ; \sigma\right)=U_{i}^{r p}\left(\neg R, s^{r p} ; \sigma\right)
$$

and

$$
\gamma_{i}^{r p}\left(s^{r p}\right)=1(0) \Longrightarrow U_{i}^{r p}\left(R, s^{r p} ; \sigma\right) \geq(\leq) U_{i}^{r p}\left(\neg R, s^{r p} ; \sigma\right) .
$$

To generalize Definition 1 to mixed strategies, say equilibrium $\sigma$ is a minority-rights equilibrium if for all irreducible absorbing sets $S$ with respect to $\sigma$ and all $s \in S$, if $\left(x^{\prime}, r^{\prime}\right) \in \mathrm{S}_{\sigma}^{\tilde{\pi}}(s)$, then $r^{\prime}=R$. With these primitives, we now prove the claim.

Existence is by construction. Consider a strategy profile $\sigma$ as in the proof of Proposition 1(Expanded)(2), except the majority leaders play the following mixed rule proposal strategy

$$
\gamma_{i}^{r p}\left(s^{r p}\right)=\left\{\begin{array}{ll}
1, & \text { if } \mu=i \\
\gamma, & \text { if } \mu \neq i
\end{array},\right.
$$


where $\gamma \in(0,1)$. Note that because policies are not persistent, there is no profitable deviation in the policy stage. We must now show there is no profitable in the procedural stage. Because moderate legislators always receive (weakly) larger payoffs under the right than majority dominance, they do not have a profitable deviation from the strategy of accepting rule proposals if and only if the proposal is the minority right. Thus, it suffices to show that for the majority leaders $i=1,4$, two conditions hold: (a) $U_{i}^{r p}\left(R, s^{r p} ; \sigma\right) \geq U_{i}^{r p}\left(\neg R, s^{r p} ; \sigma\right)$ for all $s^{r p} \in S^{r p}$ such that $\mu=i$ and (b) $U_{i}^{r p}\left(R, s^{r p} ; \sigma\right)=U_{i}^{r p}\left(\neg R, s^{r p} ; \sigma\right)$ for all $s^{r p} \in S^{r p}$ such that $i \neq \mu$. By symmetry, we only need to demonstrate this for $i=1$ and $\mu=1,2$.

I first claim condition (b) implies condition (a). To see this, suppose $U_{1}^{r p}\left(R, s^{r p} ; \sigma\right)=U_{1}^{r p}\left(\neg R, s^{r p} ; \sigma\right)$ for all $s^{r p}=(\bar{r}, \mu) \in S^{r p}$ such that $\mu=2$. If $\bar{r}=R$, then the median will reject the proposal $\neg R$, so the procedural outcomes are equivalent, which implies $U_{1}^{r p}(R,(R, 2) ; \sigma)=$ $U_{1}^{r p}(\neg R,(R, 2) ; \sigma)$. For some $s^{r p}$ such that $\bar{r}=\neg R$, note that $U_{1}^{r p}(R,(\neg R, 2) ; \sigma)=-d^{2}+U_{1}^{r p}(R,(\neg R, 1) ; \sigma)$ because only the persistent rule and the current majority party determine future payoffs. Likewise, $U_{1}^{r p}(\neg R,(\neg R, 2) ; \sigma)=U_{1}^{r p}(\neg R,(\neg R, 1) ; \sigma)$. We can now write

$$
\begin{aligned}
U_{1}^{r p}(R,(\neg R, 2) ; \sigma) & =U_{1}^{r p}(\neg R,(\neg R, 2) ; \sigma) \\
& \Longleftrightarrow-d^{2}+U_{1}^{r p}(R,(\neg R, 1) ; \sigma) \\
& =U_{1}^{r p}(\neg R,(\neg R, 1) ; \sigma) \\
& \Longleftrightarrow U_{1}^{r p}(R,(\neg R, 1) ; \sigma)>U_{1}^{r p}(\neg R,(\neg R, 1) ; \sigma) \\
& \Longleftrightarrow U_{1}^{r p}(R,(R, 1) ; \sigma)>U_{1}^{r p}(\neg R,(R, 1) ; \sigma),
\end{aligned}
$$

where the last biconditional follows because the default rule does not affect the policy or the procedural outcome when leader 1 controls a unified majority.

Hence, it suffices to verify condition (b). As before, this must hold when the default procedure $\bar{r}$ includes the minority right because the median will always reject a proposal of $\neg R$, and accordingly we only consider the case when $\bar{r}=\neg R$. So we must show $U_{1}(0, \neg R, 2 ; \sigma)=$ $U_{1}(d, R, 2 ; \sigma)$. 
Because the minority right is absorbing with $\sigma$, we can write $U_{1}(d, R, 2 ; \sigma)$ using the computations from the previous result. That is,

$$
\begin{aligned}
& U_{1}(d, R, 2 ; \sigma) \\
& \quad=\frac{-d^{2}(1+(1-2 p) \delta)(1-q \delta)+2 d(1-p)(1-q) \delta-(1-p) \delta}{(1-\delta)(1+(1-2 p) \delta)} .
\end{aligned}
$$

Define the function $F:[0,1] \rightarrow \mathbb{R}$ as

$$
\begin{aligned}
F(\gamma)= & U_{1}(0, \neg R, 2 ; \sigma) \\
= & \delta\left(p\left(q U_{1}(0, R, 1 ; \sigma)+(1-q) \gamma U_{1}(d, R, 2 ; \sigma)+(1-q)(1-\gamma) F(\gamma)\right)\right. \\
& +(1-p)\left(q U_{1}(1, R, 4 ; \sigma)(1-q) \gamma U_{1}(1-d, R, 3 ; \sigma)\right. \\
& \left.\left.+(1-q)(1-\gamma) U_{1}(1, \neg R, 3 ; \sigma)\right)\right)
\end{aligned}
$$

In this equation, $U_{1}(0, R, 1 ; \sigma), U_{1}(1, R, 4 ; \sigma)$, and $U_{1}(1-d, R, 3 ; \sigma)$ can be computed using the quantities in the previous result as well.

Next, I claim that when $p<p^{-}$there exists $\gamma^{*} \in(0,1)$ such that $F\left(\gamma^{*}\right)-U_{1}(d, R, 2 ; \sigma)=0$, which proves (b) when $\gamma=\gamma^{*}$. To show this, we demonstrate that $F(0)-U_{1}(d, R, 2 ; \sigma)<0$ and $F(1)-U_{1}(d, R, 2 ; \sigma)>$ 0 and then invoke the intermediate value theorem.

Consider $F(0)$. Then, we can write $U_{1}(1, \neg R, 3 ; \sigma)$ as

$$
\begin{aligned}
U_{1}(1, \neg R, 3 ; \sigma)= & -1+\delta\left(p q U_{1}(1, R, 4 ; \sigma)+p(1-q) U_{1}(1, \neg R, 3 ; \sigma)\right. \\
& \left.+(1-p) q U_{1}(0, R, 1 ; \sigma)(1-p)(1-q) F(0)\right)
\end{aligned}
$$

Solving gives us

$$
F(0)=\delta \frac{\begin{array}{c}
-1+p+q-p q+U_{1}(1, R, 4 ; \sigma) q(1-p) \\
+U_{1}(0, R, 4 ; \sigma) q(p+(1-2 p)(1-q) \delta)
\end{array}}{1-(1-q) \delta(2 p+(1-2 p)(1-q) \delta)} .
$$

Then, we have

$$
\begin{aligned}
F(0) & -U_{1}(d, R, 2 ; \sigma)<0 \\
& \Longleftrightarrow d\left(-d+\frac{d-1}{1-(1-q) \delta}+\frac{1}{1+(1-2 p)(1-q) \delta}\right)<0 \\
& \Longleftrightarrow p<p^{-},
\end{aligned}
$$

as required. 
Next, consider $F(1)$. In this case, every leader is implementing the right in every subsequent period. Then we have

$$
F(1)-U_{1}(d, R, 2 ; \sigma)=U_{1}(0, R, 1 ; \sigma)+d^{2}-U_{1}(0, R, 1 ; \sigma)>0,
$$

as required. Now we invoke the intermediate value theorem to show that there exists a $\gamma^{*} \in(0,1)$ such that $F\left(\gamma^{*}\right)-U_{1}(1, R, 3 ; \sigma)=0$ because $F(\gamma)$ is a polynomial and therefore continuous in $\gamma$. When $\gamma=\gamma^{*}$, condition (b) holds.

Proof of Proposition 1(Expanded)(5). Existence is by construction and follows a similar logic as the proof immediately above. Consider a strategy profile $\sigma$ defined as follows. In the policy stay, legislators follow the policy strategies detailed in the proof of Proposition 3(Expanded)(2). In the procedural stage, moderate legislator's accept a rule proposal if and only if it were to implement the minority right. Finally, the majority leaders plays the following mixed procedural proposal strategy

$$
\gamma_{i}^{r p}\left(s^{r p}\right)=\left\{\begin{array}{ll}
\gamma, & \text { if } \mu=i \\
0, & \text { if } \mu \neq i
\end{array},\right.
$$

where $\gamma \in(0,1)$. As before, it suffices to show that the majority leaders do not have a profitable deviation in the procedural stage. That is, we must show that for leaders $i=1,4$, (a) $U_{i}^{r p}\left(\neg R, s^{r p} ; \sigma\right) \geq U_{i}^{r p}\left(R, s^{r p} ; \sigma\right)$, for all $s^{r p} \in S^{r p}$ such that $\mu \neq i$ and $\bar{r}=\neg R$, and (b) $U_{i}^{r p}\left(R, s^{r p} ; \sigma\right)=$ $U_{i}^{r p}\left(\neg R, s^{r p} ; \sigma\right)$ for all $s^{r p} \in S^{r p}$ such that $i=\mu$. By symmetry, we only need to demonstrate this for $i=1$ and $\mu=1,2$.

Next, I claim the condition (b) implies condition (a). To see this, assume (b). Then we have

$$
\begin{aligned}
U_{1}^{r p}(\neg R,(\neg R, 1) ; \sigma) & =U_{1}^{r p}(R,(\neg R, 1) ; \sigma) \\
& \Longleftrightarrow U_{1}^{r p}(\neg R,(\neg R, 1) ; \sigma) \\
& >-d^{2}+U_{1}^{r p}(R,(\neg R, 1) ; \sigma) \\
& \Longrightarrow U_{1}^{r p}(\neg R,(\neg R, 2) ; \sigma)>U_{1}^{r p}(R,(\neg R, 2) ; \sigma),
\end{aligned}
$$

and $U_{1}^{r p}\left(\neg R,\left(x_{0}, R, 2\right) ; \sigma\right)=U_{1}^{r p}\left(R,\left(x_{0}, R, 2\right) ; \sigma\right)$ because the median always rejects a proposal of $\neg R$ when the default rule is $R$. Hence, we only need to verify condition $(b)$ holds. The remainder of the proof follows the argument in the proof for Proposition 1(Expanded)(4). 


\section{Proof of Proposition 2}

In this section, we prove the comparative statics in Proposition 2. Note that differentiation of $p^{+}$and $p^{-}$prove the first results. We prove the remaining one.

Proof of Proposition 2(2). Fix some $p \in(0,1)$. By Proposition 2, a minority-rights equilibrium exists if

$$
p \leq p^{+} \Longleftrightarrow p \leq \frac{2-d(1+(1-q) \delta)}{2-2 d(1-q) \delta} .
$$

As $d$ converges to $0, p^{+}$converges to 1 , which establishes the desired result.

\section{Proof of Proposition 3}

In this section, we first derive expected utilities for a more general state space. We then prove Proposition 3.

In this version, the state spaces take the following form:

\begin{tabular}{ll}
\hline Decision node $g$ & State $s^{g} \in S^{g}$ \\
\hline Rule proposal $(r p)$ & $(\bar{x}, \bar{r}, \mu)$ \\
Rule choice $(r c)$ & $\left(\bar{x}, \bar{r}, \mu, r^{p}\right)$ \\
Policy proposal $(p p)$ & $(\bar{x}, r, \mu)$ \\
Amendment proposal $(a p)$ & $\left(\bar{x}, \mu, x^{p}\right)$ \\
Policy choice with right $(m c)$ & $\left(\bar{x}, \mu, x^{p}, x^{a}\right)$ \\
Policy choice without right $(M p)$ & $\left(\bar{x}, \mu, x^{p}\right)$ \\
\hline
\end{tabular}

which just replicates Table 1 with persistent rules and policies. When $\bar{r}_{t}=\neg R$ and $\bar{x}_{t}=\bar{x}_{t-1}$ for all periods $t \geq 1$, legislator $i$ 's expected utility from passing policy $x$ with current rule $r$ and median $\mu$ take the form

$$
\begin{aligned}
& U_{i}(x, r, \mu ; \sigma) \\
& \quad=u_{i}(x)+\delta \sum_{\mu^{\prime} \in N} \pi\left(\mu^{\prime} \mid \mu\right) U_{i}\left(x^{r p}\left(x, \neg R, \mu^{\prime} ; \sigma\right), r^{r p}\left(x, \neg R, \mu^{\prime} ; \sigma\right), \mu^{\prime} ; \sigma\right) .
\end{aligned}
$$

Using this setup, it is straightforward to define $U_{i}^{g}\left(a, s^{g} ; \sigma\right)$, or $i^{\prime}$ expected utility of taking action $a \in A^{g}$ at decision node $g$ in state $s^{g}$. We now prove Proposition 3. 
Proof of Proposition 3. Consider an equilibrium $\sigma$ such that $\sigma_{i}^{r p}\left(s^{r p}\right)=$ $R$ for some leader $i$ and initial state $s^{r p}=(\bar{x}, \bar{r}, \mu)$. Then $x^{r p}\left(s^{r p} ; \sigma\right)=x$ and $r^{r p}\left(s^{r p} ; \sigma\right)=r$ are the policy and procedural outcomes of this state, respectively. Construct profile $\bar{\sigma}$ as follows. At $s^{r p}, i$ proposes $\neg R$ as the rule, which will be the rule governing the stage. In the policy stage, $i$ uses strategy $\sigma_{i}^{p p}(\bar{x}, \neg R, \mu)=x$, i.e., he proposes the policy outcome of the state under the original equilibrium. Finally, the median accepts the proposal $x$ when the status quo is $\bar{x}$ and rule $\neg R$. All other strategies remain the same.

I claim that $\bar{\sigma}$ is an equilibrium. To see this, first note that $x^{r p}\left(s^{r p} ; \bar{\sigma}\right)=x$ and $r^{r p}\left(s^{r p} ; \bar{\sigma}\right)=r$. By the construction of $\bar{\sigma}$,

$$
\begin{aligned}
U_{i}(x, \neg R, \mu ; \bar{\sigma})= & u_{i}(x)+\delta \sum_{\mu^{\prime} \in N} \pi\left(\mu^{\prime} \mid \mu\right) U_{i}\left(x^{r p}\left(x, \neg R, \mu^{\prime} ; \bar{\sigma}\right),\right. \\
& \left.\quad r^{r p}\left(x, \neg R, \mu^{\prime} ; \bar{\sigma}\right), \mu^{\prime} ; \bar{\sigma}\right) \\
= & U_{i}(x, R, \mu ; \sigma),
\end{aligned}
$$

for all actors $i$ and all policies $x$ because all policy outcomes in every future state remain unchanged and the current rule does not affect future payoffs. Now suppose the median has a profitable deviation by choosing $\bar{x}$ over $x$. Then the median must prefer the status quo $\bar{x}$ to $x$, formally $U_{\mu}(\bar{x}, \neg R, \mu ; \bar{\sigma})>U_{\mu}(x, \neg R, \mu ; \bar{\sigma})$. However, if this were the case, the above string of equalities imply that $U_{\mu}(\bar{x}, R, \mu ; \sigma)>U_{\mu}(x, R, \mu ; \sigma)$, which means that the median has a profitable deviation under the original equilibrium, a contradiction.

Now suppose leader $i \in\{1,4\}$ has a profitable deviation in the policy stage. That is

$$
\left.x \notin \arg \max _{z \in \mathbb{R}} U_{i}^{p p}(z,(\bar{x}, \neg R, \mu) ; \bar{\sigma})\right) .
$$

Consider some $x^{p} \in \mathbb{R}$ such that $U_{i}^{p p}\left(x^{p},(\bar{x}, \neg R, \mu) ; \sigma\right)>U_{i}^{p p}(x,(\bar{x}, \neg R$, $\mu) ; \sigma)$. Let $x^{\prime}$ denote the policy outcome associated with proposal $x^{p}$ given $\bar{\sigma}$. Then

$$
\begin{gathered}
U_{i}^{p p}\left(x^{p},(\bar{x}, \neg R, \mu) ; \bar{\sigma}\right)>U_{i}^{p p}(x,(\bar{x}, \neg R, \mu) ; \bar{\sigma}) \\
\quad \Longrightarrow U_{i}\left(x^{\prime}, \neg R, \mu ; \bar{\sigma}\right)>U_{i}(x, \neg R, \mu ; \bar{\sigma}) \\
\quad \Longrightarrow U_{i}\left(x^{\prime}, \neg R, \mu ; \sigma\right)>U_{i}(x, \neg R, \mu ; \sigma) \\
\quad \Longrightarrow U_{i}\left(x^{\prime}, \neg R, \mu ; \sigma\right)>U_{i}(x, r, \mu ; \sigma)
\end{gathered}
$$




$$
\begin{aligned}
& \Longrightarrow U_{i}^{r p}\left(\neg R, s^{r p} ; \sigma\right)>U_{i}^{r p}\left(R, s^{r p} ; \sigma\right) \\
& \Longrightarrow U_{i}^{r p}\left(\neg R, s^{r p} ; \sigma\right)>U_{i}^{r p}\left(\sigma^{r p}\left(s^{r p}\right), s^{r p} ; \sigma\right)
\end{aligned}
$$

The first implication follows by the definition of $x^{\prime}$ and $\bar{\sigma}$. The second implication follows from the string of equalities in the preceding paragraph. The third implication follows because rules are not persistent and do not affect future policy outcomes or current payoffs, so $U_{i}(x, \neg R, \mu ; \sigma)=U_{i}(x, R, \mu ; \sigma)$. The fourth and final implication follows from the optimality of $i$ 's play at all decision nodes and the definition of $\sigma$. Specifically, because $\sigma$ is an equilibrium, $U_{i}^{r p}\left(\neg R, s^{r p} ; \sigma\right) \geq U_{i}\left(x^{\prime}, \neg R, \mu ; \sigma\right)$. However, the last inequality contradicts the assumption that $\sigma$ is an equilibrium.

A similar argument rules out deviations in the procedural stage. For one to exist, with the right, there must exist a proposal $x^{p}$ such that $U_{i}^{p p}\left(x^{p},(\bar{x}, R, \mu) ; \bar{\sigma}\right)>U_{i}^{p p}(x,(\bar{x}, \neg R, \mu) ; \bar{\sigma})$, which would imply that $x^{p}$ produces policy $x^{\prime}$ were $U_{i}\left(x^{\prime}, R, \mu ; \sigma\right)>U_{i}(x, \neg R, \mu ; \sigma)$. But the rules are not persistent and do not directly affect utility outcomes. Therefore, $U_{i}\left(x^{\prime}, \neg R, \mu ; \sigma\right)>U_{i}(x, \neg R, \mu ; \sigma)$. By the argument above, this leads to a contradiction.

\section{References}

Alesina, A. (1988), "Credibility and Policy Convergence in a Two-Party System with Rational Voters", American Economic Review, 78(4), 796-805.

Banks, J. S. and J. Duggan (2006), "A Social Choice Lemma on Voting over Lotteries with Applicatons to a Class of Dynamic Games", Social Choice and Welfare, 26(2), 285-304.

Baron, D. P. (1996), "A Dynamic Theory of Collective Goods Programs", American Political Science Review, 90(2), 316-30.

Binder, S. A. (1996), "The Partisan Basis of Procedural Choice: Allocating Parliamentary Rights in the House, 1789-1990", American Political Science Review, 90(1), 8-20.

Binder, S. A. (1997), Minority Rights and Majority Rule: Partisanship and the Development of Congress, Cambridge: Cambridge University Press. 
Binder, S. A. and S. S. Smith (1997), Politics or Principle: Filibustering in the United States Senate, Washington, DC: Brookings Institution Press.

Campbell, A. C., G. W. Cox, and M. D. McCubbins (2002), "Agenda Power in the U.S. Senate, 1877-1986", in Party Process and Political Change in Congress: New Perspectives on the History of Congress, ed. D. W. Brady and M. D. McCubbins, Stanford: Stanford University Press.

Clausen, A. and C. Wilcox (1987), "Policy Partisanship in Legislative Leadership, Recruitment and Behavior", Legislative Studies Quarterly, 12(2), 243-64.

Cotton, C. (2012), "Dynamic Legislative Bargaining with Endogenous Agenda Setting Authority", Unpublished manuscript.

Cox, G. W. and M. D. McCubbins (1993), Legislative Leviathan, Cambridge: Cambridge University Press.

Cox, G. W. and M. D. McCubbins (2005), Setting the Agenda, Cambridge: Cambridge University Press.

Diermeier, D., C. Prato, and R. Vlaicu (2013), "Procedural Choice in Majoritarian Organizations", American Journal of Political Science, forthcoming.

Diermeier, D. and R. Vlaicu (2011), "Parties, Coalitions, and their Internal Organization of Legislatures", American Political Science Review, 105(2), 359-80.

Dion, D. (1997), Turning the Legislative Thumbscrew, Ann Arbor: The University of Michigan Press.

Dixit, A., G. M. Grossman, and F. Gul (2000), "The Dynamics of Political Compromise", Journal of Political Economy, 108(3), 53168.

Duggan, J. and T. Kalandrakis (2012), "Dynamic Legislative Policy Making", Journal of Economic Theory, 147(5), 1653-88.

Eguia, J. and K. A. Shepsle (2013), "Endogenous Assembly Rules, Senior Agenda Power, and Incumbency Advantage", Unpublished manuscript.

Fenno, R. F. (1973), Congressmen in Committees, Boston: Brown and Company Inc.

Fox, J. (2006), "Legislative Cooperation among Impatient Legislators", Journal of Theoretical Politics, 18(1), 68-97. 
Gailmard, S. and J. A. Jenkins (2007), "Negative Agenda Control in the Senate and House: Fingerprints of Majority Party Power", Journal of Politics, 69(3), 689-700.

Gilligan, T. W. and K. Krehbiel (1987), "Collective Decisionmaking and Standing Committees: An Informational Rationale for Restrictive Amendment Procedures", Journal of Law, Economics and Organization, 3(2), 287-335.

Grofman, B., W. Koetzle, and A. J. McGann (2002), "Congressional Leadership 1965-1996: A New Look at the Extremism Versus Centrality Debate", Legislative Studies Quarterly, 27(1), 87-105.

Huber, J. D. (1996), Rationalizing Parliament, New York: Cambridge University Press.

Jeon, J. S. (2015), "Them as Has, Gets: A Dynamic Model of Endogenous Proposal Power", Unpublished manuscript.

Kalandrakis, T. (2004), "A Three-Player Dynamic Majoritarian Bargaining Game", Journal of Economic Theory, 116(2), 294-322.

Kalandrakis, T. (2006), "Proposal Rights and Political Power", American Journal of Political Science, 50(2), 441-8.

King, D. C. and R. J. Zeckhauser (2002), "Punching and CounterPunching in the U.S. Congress: Why Party Leaders Tend to be Extremists", Paper presented at the Conference on Leadership 2002: Bridging the Gap Between Theory and Practice, The Center for Public Leadership, Cambridge, MA.

Koger, G. (2010), Filibustering: A Political History of Obstruction in the House and Senate, Chicago: University of Chicago Press.

Krehbiel, K. (1991), Information and Legislative Organization, Ann Arbor: University of Michigan Press.

Krehbiel, K. and A. Meirowitz (2002), "Minority Rights and Majority Power: Theoretical Consequences of the Motion to Recommit", Legislative Studies Quarterly, 27(2), 191-217.

Krehbiel, K., A. Meriowitz, and A. E. Wiseman (2015), "A Theory of Competitive Partisan Lawmaking", Political Science Research and Methods, forthcoming.

Lazarus, J. and A. Steigerwalt (2009), "Different Houses: The Distribution of Earmarks in the US House and Senate", Legislative Studies Quarterly, 34(3), 347-73. 
Mattson, I. (1995), "Private Members' Intiatives and Amendments", in Parliaments and Majority Rule in Western Europe, ed. H. Döring, Frankfurt: Campus Verlag.

McKelvey, R. D. (1976), "Intransitivities in Multidimensional Voting Models and Some Implications for Agenda Control", Journal of Economic Theory, 12(3), 472-82.

McKelvey, R. D. and R. Riezman (1992), "Seniority in Legislatures", American Political Science Review, 86(4), 951-65.

Rasch, B. (1995), "Parliamentary Voting Procedures", in Parliaments and Majority Rule in Western Europe, ed. H. Döring, Frankfurt: Campus Verlag.

Roberts, J. M. (2005), "Minority Rights and Majority Power: Conditional Party Government and the Motion to Recommit in the House", Legislative Studies Quarterly, 30(2), 219-34.

Romer, T. and H. Rosenthal (1978), "Political Resource Allocation, Controlled Agendas, and the Status Quo", Public Choice, 33(4), $27-43$.

Roust, K. A. (2005), "Minority Rights in Majoritarian Institutions", $\mathrm{PhD}$ thesis, California Institute of Technology.

Schickler, E. (2000), "Institutional Change in the House of Representatitves, 1867-1998: A Test of Partisan and Ideological Power Balance Models", American Political Science Review, 94(2), 269-88.

Shepsle, K. A. and B. R. Weingast (1987), "The Institutional Foundations of Committee Power", The American Political Science Review, 81(1), 85-104.

Smith, S. S. (2007), Party Influence in Congress, Cambridge University Press.

Smith, S. S., I. Ostrander, and C. M. Pope (2013), "Majority Party Power and Procedural Motions in the US Senate", Legislative Studies Quarterly, 38(2), 205-36.

Suk-Young Chwe, M. (1999), "Minority Rights Can Maximize Majority Welfare", American Political Science Review, 93(1), 85-97.

Wawro, G. J. and E. Schickler (2006), Filibuster: Obstruction and Lawmaking in the U.S. Senate, Princeton: Princeton University Press. 
Wolfensberger, D. R. (2007), "The Motion to Recommit in the House: The Creation, Evisceration, and Restoration of a Minority Right", in Party, Process, and Political Change in Congress, ed. D. W. Brady and M. D. McCubbins, Vol. 2, Stanford, California: Stanford University Press.

Yildirim, H. (2007), "Proposal Power and Majority Rule in Multilateral Barganing with Costly Recognition", Journal of Economic Theory, 136(1), 167-96.

Zapal, J. (2011), "Explicit and Implicit Status-quo Determination in Dynamic Bargaining: Theory and Application to FOMC Directive", Unpublished manuscript. 